Pobreza por escasez de ingresos y por falta de tiempo en la Argentina

\author{
Carla Arévalo \\ Tesis de Maestría \\ Maestría en Economía \\ Universidad Nacional de La Plata
}

Directora de Tesis: Adriana Conconi

Co-directora de Tesis: Roxana Maurizio

Fecha de defensa:

Códigos JEL: J22 D31 


\title{
Pobreza por escasez de ingresos y por falta de tiempo en la Argentina
}

\section{Resumen}

Este trabajo incorpora la dimensión temporal a la medición de pobreza utilizando la metodología LIMTIP sobre datos de la Encuesta de Trabajo no Remunerado y Uso del Tiempo relevada por el Instituto Nacional de Estadísticas y Censos (INDEC) en el año 2013. Se trata de una metodología que intenta abordar de manera más integral el análisis del bienestar generando información sobre la interrelación entre el mercado de trabajo, la estructura demográfica de los hogares y las políticas sociales con una perspectiva de género. Entre los hallazgos más relevantes se puede mencionar que la pobreza LIMTIP incide entre los hombres y las mujeres a niveles muy similares. Además, el sexo del jefe de hogar no constituye un determinante de la pobreza LIMTIP. Por lo tanto, la evidencia sugiere que la pobreza que afecta a mujeres o a hogares comandados por ellas tiene fuentes diferentes más allá de su condición de mujer.

Palabras clave: Uso del tiempo, Pobreza, Género, Trabajo decente.

\begin{abstract}
This study incorporates a temporal dimension into poverty measurement using the LIMTIP methodology on data from the Unpaid Work and Time Use Survey collected by the National Institute of Statistics and Census (INDEC) in 2013. This analysis provides information about the relationship between the labor market, the demographic structure of households and social policies under a gender perspective. Among the most important findings we can mention that LIMTIP poverty affects men and women at very similar levels. Besides, the gender of the household head is not a determinant of poverty LIMTIP. Therefore, the evidence suggests that poverty that affects women or households led by them has different drivers beyond their gender.
\end{abstract}

Keywords: Time use, Poverty, Gender, Decent work. 
Pobreza por escasez de ingresos y por falta de tiempo en la Argentina

\section{Índice}

Capítulo I Introducción 1

Capítulo II Marco teórico 3

Capítulo III Revisión de la literatura 5

$\begin{array}{lll}\text { Capítulo IV Datos } & 10\end{array}$

Breve reseña de las encuestas de uso del tiempo en la Argentina

Medición de la pobreza LIMTIP 13

Aplicación empírica 16

Análisis condicional 20

$\begin{array}{lll}\text { Capítulo VI Resultados } & 21\end{array}$

Medición de la pobreza LIMTIP

Análisis condicional 26

Capítulo VII Consideraciones Finales 30

Capítulo VIII Referencias Bibliográficas 32 


\section{Introducción}

El propósito de este estudio es analizar el bienestar de la población argentina intentando responder a las preguntas ¿quiénes son pobres? y ¿por qué son pobres? Para ello se ha seleccionado al ingreso como variable focal pero también se incorpora el tiempo como un componente importante, aunque generalmente omitido, del bienestar. La combinación de ingreso y tiempo en una medida de pobreza permite identificar tanto a las personas que carecen de recursos para satisfacer ciertas necesidades básicas, como a aquellas con déficit de tiempo e imposibilidad de adquirir sustitutos en el mercado para menguar ese déficit. Como suele suceder bajo un análisis de pobreza monetaria, serán identificados como pobres los trabajadores mal remunerados, pero también los que escapan de la pobreza monetaria trabajando largas horas sumidos en déficit de tiempo y, como es habitual, a las mujeres en hogares cuyo ingreso familiar es insuficiente, pero también a aquellas con un ingreso laboral adecuado pero que enfrentan una "doble jornada": una en el mercado de trabajo y otra en el hogar.

El poder de identificación de esta medida generalizada brinda una mejor comprensión del estado de bienestar de la población con implicancias en un amplio rango de áreas de políticas desde las tendientes a aliviar la pobreza hasta las que regulan el empleo. Estas implicancias son coherentes con los objetivos y metas propuestos por Naciones Unidas para la agenda 2015-2030 sobre pobreza, trabajo decente e igualdad de género, entre los cuales se puede mencionar: el objetivo 1 para el cual resulta imperante "poner fin a la pobreza en todas sus formas en todo el mundo", el 5.4 que sugiere "reconocer y valorar los cuidados no remunerados y el trabajo doméstico no remunerado mediante la prestación de servicios públicos, la provisión de infraestructuras y la formulación de políticas de protección social, así como mediante la promoción de la responsabilidad compartida en el hogar y la familia, según proceda en cada país", y el 5.5 que plantea "velar por la participación plena y efectiva de las mujeres y la igualdad de oportunidades de liderazgo a todos los niveles de la adopción de decisiones en la vida política, económica y pública" (NU, 2015).

Este estudio se basa en la aplicación de métodos cuantitativos en la Encuesta sobre Trabajo no Remunerado y Uso del Tiempo relevada por el Instituto Nacional de Estadísticas y Censos de la República Argentina (INDEC) en el año 2013. Además de un primera aproximación a través de la construcción de perfiles con variables de interés, 
se estima un modelo probit con intención de detectar los factores asociados de mayor impacto sobre la condición de pobreza.

Aun cuando los estudios sobre empleo del tiempo tuvieron un fuerte impulso con el análisis de la desigualdad entre géneros, se ha encontrado evidencia de que la pobreza incide entre los hombres y las mujeres a niveles muy similares. Además, en un análisis condicional de la pobreza LIMTIP el sexo del jefe de hogar pierde significancia estadística cuando se incorporan controles sobre el tipo de ocupación y la educación del jefe. Por lo tanto se concluye que el patrón de mayor incidencia de pobreza LIMTIP en hogares con jefatura femenina respondería a las dotaciones con que cuentan las mujeres y no a su condición de mujer.

Este trabajo de tesis está organizado de la siguiente manera: en el próximo capítulo se explica el marco teórico que fundamenta la incorporación del tiempo a la medición de pobreza; en la sección III se comentan antecedentes sobre estudios de uso y pobreza en tiempo con diferentes metodologías y para diferentes países; en la sección IV se presentan los datos utilizados y una breve reseña general de las encuestas sobre empleo del tiempo y, en particular, las realizadas en la Argentina; la sección V describe las metodologías empleadas en la medición y en el análisis de la pobreza; los resultados obtenidos son presentados en la sección VI; finalmente en la sección VII se presentan las consideraciones finales del trabajo y recomendaciones de política.

\section{Marco teórico}

Este trabajo de investigación explota un supuesto implícito presente en la construcción de la línea de pobreza absoluta en las mediciones tradicionales de la pobreza: disponibilidad de una persona que puede trabajar a tiempo completo en el hogar (o disponibilidad de la cantidad de horas necesarias para la producción doméstica). Según un grupo de expertos en estadísticas de pobreza, las líneas de pobreza absoluta reflejan el costo de adquirir una canasta de elementos esenciales para alcanzar los umbrales absolutos de satisfacción de ciertas necesidades básicas (Grupo de Río, 2007). A partir de esta definición, se entiende que construir una línea de pobreza requiere, primero, establecer cuáles son esas necesidades básicas a satisfacer. Ravallion (1999) define a las necesidades básicas como un mínimo normativo socialmente determinado para evitar la pobreza. Es decir, son criterios sobre los cuales no existe un consenso general y que 
pueden variar según el contexto. Con todo, se puede afirmar que la necesidad que supera toda discusión es la ingesta de alimentos.

Grupo de Río (2007) explica que para obtener una línea de pobreza alimentaria se puede elegir entre dos alternativas: una consiste en construir una canasta de alimentos explícita y luego asignarle un precio; y la otra, en tomar el costo por caloría sin detallar el contenido de la canasta. Sea cual fuere el camino elegido, en ningún caso el tiempo necesario para transformar esos productos en comida es tenido en cuenta. Esa omisión cobra todavía mayor relevancia ya que el objetivo deseado es obtener una canasta mínima, y, como bien explica Vickery (1977), mientras menor es el costo del plan de comida, mayor es la necesidad de habilidades y tiempo para la preparación.

\section{Figura 1}

\section{Umbral de pobreza para un hogar}

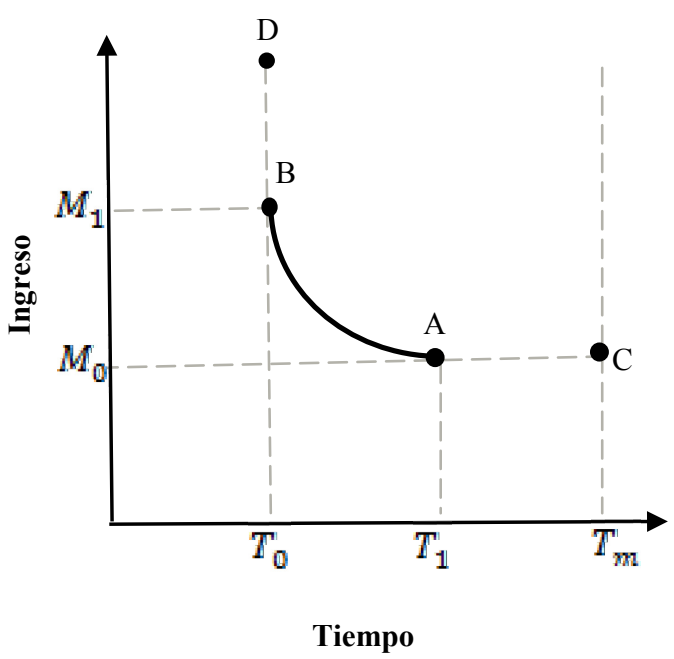

Fuente: Tomado de Vickery (1977: 28).

Pongamos una situación hipotética para clarificar la idea anterior de intercambio entre el tiempo y el costo de un plan de comida. Supongamos que para obtener un kilogramo de pan se necesitan \$40 y 5 minutos para llegar a la panadería más cercana, o se pueden comprar los insumos necesarios para preparar la misma cantidad de pan con la mitad del dinero, pero destinando más de una hora a la preparación. Este ejemplo sugiere que los recursos de un hogar se determinan tanto por sus activos como por la cantidad de horas adulto disponibles para ganar dinero en el mercado o para producir bienes de consumo y servicios fuera del mercado. En esta dirección, Vickery (1977) propone fijar un umbral de pobreza como se muestra en la Figura 1, una isocuanta que representa diferentes combinaciones de tiempo e ingreso. 
Utilizando las estimaciones de pobreza tradicional se identifica como pobre a aquellas familias que se encuentren por debajo de la línea horizontal $M_{0} C$, o, lo que es lo mismo, aquellas cuyo ingreso no alcanza el umbral $M_{0}$. En cambio, desde la perspectiva generalizada, la identificación se construye a partir de dos supuestos: 1) alcanzar el umbral de pobreza requiere un mínimo de tiempo $\left(T_{0}\right)$ independientemente del ingreso disponible, y un mínimo de ingreso $\left(M_{0}\right)$ independientemente de la cantidad de tiempo disponible. Así, un hogar será considerado pobre si su tiempo o ingreso caen por debajo de esos niveles; 2) $M_{0}$ y $T_{0}$ no son suficientes para alcanzar un estándar de bienestar que esté fuera de la pobreza. Es decir que, si sólo se tiene $T_{0}$ de tiempo ( $M_{0}$ de ingreso) disponible, se necesita $M_{1}\left(T_{1}\right)$ para no ser considerado pobre.

Con estos elementos estamos en condiciones de establecer que los puntos sobre una isocuanta de pobreza son combinaciones de tiempo e ingreso suficientes para alcanzar un estándar de bienestar mínimo fuera de la pobreza. Por lo tanto, los hogares identificados como pobres son todos aquellos que están por debajo de la curva CABD. Para entender mejor el proceso de identificación veamos que a los hogares identificados como pobres según la perspectiva tradicional (aquellos con ingresos menores a $M_{0}$ ), se les agregan aquellos cuyo ingreso supera $M_{0}$ (la línea de pobreza monetaria), pero que tienen una disponibilidad de tiempo limitada en relación al tiempo requerido por el hogar para reproducirse. Esta es la definición de pobreza que sustenta el procedimiento de medición que se desarrolla en este estudio.

\section{Revisión de la literatura}

Se desprende de la sección anterior que el tiempo es un componente importante, aunque generalmente omitido, en la medición de la pobreza. Este componente es considerado por Martínez (2005) como la pieza que faltaba para analizar la producción de bienestar. Esta autora considera que a partir del análisis del uso del tiempo es posible rescatar el rol de las familias como productoras y articuladoras del bienestar. Por su parte, Esquivel (2014) indica que el trabajo de las familias (doméstico y de cuidados) "expande el ingreso nacional, y por lo tanto el bienestar". Las familias son productoras porque realizan actividades, al igual que los mercados y los Estados, para crear bienes y servicios. A la vez, asignan los recursos procedentes del mercado, de las políticas públicas y de las mismas familias, y por eso son articuladoras. 
El Levy Economics Institute of Bard College ha desarrollado una nueva forma de estimar pobreza considerando tanto la insuficiencia de ingresos como la escasez de tiempo. Las medidas que propone el instituto se conocen como medidas LIMTIP por sus siglas en inglés (Levy Institute's Innovative Measure of Time and Income Poverty) y son varios los documentos que explican los procedimientos de estimación. Por ejemplo, Materson (2001, 2012 y 2014) describe el procedimiento para unir bases de hogares con bases de uso del tiempo para los casos de Argentina, Chile, México y Corea del Sur, obtiene uniones de alta calidad dadas las fuentes de datos disponibles y realiza algunos ejercicios de simulación.

Burchardt (2008) también utiliza la metodología LIMTIP y revisa en detalle las políticas sociales que afectan a las canastas de tiempo e ingreso, especialmente en familias de ingresos bajos, para el caso del Reino Unido. Resulta interesante como la autora se vale, además de las estimaciones, de un análisis de tipo cualitativo para ilustrar las estrategias que adoptan las personas para lidiar con las tensiones entre el tiempo y el dinero. Particularmente, una de las mujeres entrevistadas, siendo madre soltera de un niño de 3 años, dijo: "El punto es pasar tiempo con mi hijo, así que no me importa ser pobre por un tiempo", dejando entrever la necesidad de resignar un ingreso potencial al priorizar la crianza personal de su hijo.

El antecedente más directo de este trabajo de tesis es el estudio de Esquivel (2014), quien aplica la metodología en la Ciudad Autónoma de Buenos Aires (CABA) con datos de 2005. Su estudio indica que la importancia de medir pobreza en tiempo radica en "reconocer que el bienestar económico de los hogares y las personas no depende solamente del trabajo remunerado, sino también del trabajo doméstico y del cuidado no remunerado". Además, resalta que el diseño de políticas debe avanzar no sólo sobre la mera generación de empleo, sino más bien sobre la generación de empleo decente. Puntualmente, explica que los segmentos más vulnerables podrán superar los déficits de tiempo en la medida en que reciban salarios dignos, se regulen las jornadas laborales, se adopten medidas de protección social y de conciliación entre la vida laboral y familiar, y se provean servicios de cuidado infantil.

Las estimaciones de Esquivel (2014) resultan orientadoras y exhiben ciertos patrones de interés. Según la autora en el año 2005, CABA registró una incidencia de la pobreza monetaria de $8,8 \%$, mientras que al incorporar los déficits de tiempo a la medición ese 
nivel casi se duplica (15,9\% de la población). La diferencia indica que existen personas sobre las que no se focalizan políticas porque no son consideradas pobres en términos monetarios, pero que no disponen del tiempo suficiente para cubrir las tareas domésticas y de cuidado mínimas ni del nivel de ingreso suficiente para adquirir sustitutos.

Esquivel (2014) agrega que la pobreza en tiempo afecta más a los hogares familiares ${ }^{1}, \mathrm{y}$ todavía más a aquellos con presencia de menores y monoparentales con jefatura femenina. Además, tras una simulación que deriva en una situación de pleno empleo, obtiene una caída radical en la pobreza oficial que casi desaparece, mientras que la pobreza corregida por déficits de tiempo disminuye pero persiste. Este resultado muestra que las políticas de creación pura de empleo tienen limitaciones $\mathrm{y}$, aunque parecen ser efectivas para reducir la pobreza monetaria, no son suficientes para garantizar un nivel de bienestar considerado mínimo (aquél alcanzado una vez cubierta la canasta básica total y los requerimientos mínimos de tiempo para la reproducción del hogar).

En la literatura también se encuentran otras formas de medición de la pobreza en tiempo. Se puede mencionar la utilización de la noción de pobreza relativa, por la cual se considera pobre en tiempo a todo aquél que tenga menos tiempo libre que cierto umbral relativo al resto de la población, como es el caso de Merz y Rathjen (2014) quienes utilizan como umbral el $60 \%$ de la mediana del tiempo de ocio personal. También se ha incorporado al tiempo como una privación más en las mediciones de pobreza multidimensional. Utilizando este mecanismo, Carbajal (2011) encuentra que al incorporar la privación de tiempo, la pobreza multidimensional se incrementa y que esta privación contribuye a la pobreza total alrededor de un $10 \%$.

Por otra parte, el análisis del uso de tiempo ha tomado relevancia entre los estudios de género ya que en general se encuentran fuertes disparidades en la distribución de las tareas domésticas y de cuidado. Según Martínez (2005), las familias participan de las actividades productivas con una marcada división por género. Tras analizar la evidencia de siete países latinoamericanos (República Dominicana/1995, Nicaragua/1998, Guatemala/2000, Cuba/2001, México/2002, Uruguay/2003 y Costa Rica/2004), la autora concluye que, en general, las mujeres destinan varias veces más horas que los hombres a este tipo de actividades. Para Esquivel (2014), la situación en la Capital

\footnotetext{
${ }^{1}$ Todos los hogares son considerados hogares familiares, excepto los hogares unipersonales o aquellos en los que no existe parentesco entre sus miembros.
} 
Federal de la República Argentina (la Ciudad Autónoma de Buenos Aires, CABA) en 2005 coincide con lo anterior ${ }^{2}$, y agrega que la brecha se profundiza para las madres de niños pequeños y para las mujeres ocupadas.

En lo que respecta a las actividades de articulación (por ejemplo, hacer compras o realizar trámites), la distribución es menos clara. Aparentemente, existe cierta relación entre la detentación de autoridad dentro del hogar y las actividades de articulación. Generalmente son los hombres quienes realizan este tipo de tareas, especialmente aquellas que implican manejar dinero. Sin embargo, más que una cuestión sexista parece ser una cuestión de poder, ya que, por ejemplo, en Nicaragua no se verifica brecha alguna en el tiempo destinado a las tareas de articulación entre hombres y mujeres jefes de hogar, quienes se presume tienen un grado de autoridad similar por su condición de jefatura (Martínez, 2005).

En las últimas décadas la participación femenina en el mercado de trabajo ha ido aumentando, sin embargo ese aumento no parece haber sido compensado con menos horas en el trabajo no remunerado. Según Martínez (2005) incluso en hogares con doble proveedor son las mujeres las que mantienen la mayor carga de las tareas domésticas. Es decir que, aunque tanto la mujer como el hombre trabajen fuera del hogar (aproximadamente la misma cantidad de horas), es la mujer la que mantiene la mayor carga horaria en la producción doméstica. Esta situación se verifica, por ejemplo, en los países de Costa Rica, Guatemala y Nicaragua caracterizados por una alta demanda de cuidado generada por los altos niveles de fecundidad, combinada con una escasa inversión social. También en la Argentina, particularmente en CABA en el año 2005, año en que la tasa de pobreza en tiempo de mujeres ocupadas superó a la de los hombres con la misma condición de ocupación (Antonopoulos et al., 2016).

Algunos estudios como el de Bardasi y Wodon (2006) y el de Öneş et al. (2013) reportan las diferencias en el uso del tiempo de hombres y mujeres según condición de pobreza y zonas de residencia rural o urbana. Öneş et al. (2013) encuentran que, en Turquía en el año 2006, las mujeres destinaban mayor cantidad de horas al trabajo, sumando tareas remuneradas y no remuneradas, que lo hombres. Entre las mujeres, el trabajo doméstico explicaba el $80 \%$ del total de horas de trabajo, mientras que los

\footnotetext{
${ }^{2}$ Varios son los trabajos que arrojan evidencia en la misma dirección. Se puede consultar McGinnity y Russell (2008) para el caso de Irlanda en 2005 y Bloemen (2010) con evidencia para 15 países europeos, entre otros.
} 
hombres dedicaban sólo el $10 \%$ de su tiempo de trabajo en tareas domésticas. Este patrón se registra tanto para pobres por ingreso como para no pobres. También muestran que las mujeres pobres tenían más horas de trabajo total que las no pobres, mientras que la relación entre varones pobres y no pobres se torna en sentido opuesto. Para dar una idea de la brecha entre áreas de residencia los autores explican que las mujeres pobres en zona rural registraban 5,5 veces más horas de trabajo remunerado que las mujeres pobres en zona urbana.

En términos de pobreza de tiempo, Bardasi y Wodon (2006) muestran que en Guinea 2002-2003, las mujeres eran más pobres que los hombres. Además, las mujeres en áreas rurales eran más pobres que sus pares en áreas urbanas, aunque ocurría lo contrario entre los hombres. El aporte de este estudio en pobreza de tiempo en niñez merece ser destacado. Si bien las niñas también son más pobres que los varones como ocurre con los adultos, la brecha entre áreas de residencia es mucho más alta. Entre los niños de la ciudad la tasa de pobreza en tiempo es de $7,7 \%$ mientras que en la zona rural ese valor asciende al 40,9\%; entre las niñas la incidencia es mayor: 20,4 en áreas urbanas y 56,9\% en zona rural.

En los estudios sobre uso del tiempo con perspectiva de género en ciudades argentinas también se encuentra una situación de desventaja para el género femenino. En CABA (2005) y en la ciudad de Rosario en Santa Fe (2010), los hombres duplican la cantidad de horas que las mujeres destinan al trabajo remunerado, mientras que ellas dedican tres veces más tiempo que los hombres al trabajo no remunerado (Ganem et al., 2014).

No obstante, Arévalo y Paz (2015) analizan la desigualdad de género en el uso del tiempo en CABA en el año 2005 y encuentran que mujeres y hombres de grupos comparables $^{3}$ no difieren demasiado en el número de horas que dedican al trabajo total (remunerado y no remunerado). Tampoco se observan diferencias significativas en las tasas de empleo corregidas (sumando a las horas de trabajo, las dedicadas a tareas domésticas y de cuidado). Este hallazgo resulta relevante dado que las tasas de empleo y de participación tradicionales muestran niveles muy superiores para los hombres, sin hacer visible el hecho de que las mujeres están realizando tareas que generan valor económico igual que las tareas mercantiles. Sí se pudo constatar, como en los estudios mencionados anteriormente, una marcada división del trabajo por género, ya que las

\footnotetext{
${ }^{3}$ Se considera que son grupos comparables ya que las diferencias surgen de modelos en los que se han incorporado variables sociodemográficas habituales que permiten aislar el efecto del género.
} 
mujeres son quienes principalmente asumen las tareas de cuidado de niñas, niños y adolescentes en el hogar.

Sin embargo, la división del trabajo por género no necesariamente sugiere una situación de desventaja para las mujeres. Resulta interesante mencionar el hallazgo de Martínez (2005) sobre las actividades domésticas que aparentemente las mujeres eligen. La autora encuentra evidencia en Uruguay y México que sugiere que entre las mujeres con mayor poder de decisión (aquellas con mayor nivel educativo, que no están en condiciones de pobreza o con mayores ingresos) la dedicación al cuidado de niños es mayor. Pero ocurre lo contrario con otras tareas como la preparación de alimentos. En otras palabras, cuanto más alto en la estructura social, mayor delegación y derivación del trabajo doméstico y mayor prioridad a actividades de cuidado.

\section{Datos}

Aguirre y Ferrari (2014) revisan la trayectoria de la implementación de encuestas sobre empleo del tiempo en América Latina desde el primer antecedente desarrollado en Cuba en el año 1985. Según las autoras, las estrategias de relevamiento de este tipo de encuestas se presentan en módulos asociados a una encuesta principal o a través de encuestas independientes. La elección de una u otra estrategia depende principalmente de factores políticos y técnicos vinculados al financiamiento disponible. Ambas estrategias pueden ser abordadas utilizando dos tipos de instrumentos: un diario de empleo del tiempo o una lista de actividades. El primero consiste en un registro de las actividades realizadas en un periodo de 24 horas, o de 48 horas si se agregan actividades de un día atípico (fines de semana). También es posible recolectar información a partir de una versión simplificada que consiste en registrar la duración de actividades durante un período específico, que puede o no coincidir con una jornada de 24 horas.

La lista de actividades consiste en hacer preguntas sobre la frecuencia con que se realiza una serie de actividades, y recoger información sobre el tiempo dedicado a cada una de ellas. Las autoras explican que el diario de actividades tiene como ventaja que se registran todas las actividades realizadas, hora de inicio y finalización. Es decir, que se obtiene mayor precisión y detalle que con la lista. Además, como las preguntas refieren al día anterior hay mayor grado de fiabilidad en las respuestas apelando a un esfuerzo menor de la memoria del encuestado. Finalmente, el diario permite conocer la realización de actividades simultáneas otorgando diferentes rangos de relevancia a cada 
una. Por su parte, la lista de actividades provee información específica en función de la demanda para el diseño de políticas públicas sobre problemáticas puntuales, en lugar del detalle exhaustivo de todas las actividades que realizan los encuestados.

\section{Breve reseña de las encuestas de uso del tiempo en la Argentina}

En la Argentina, se han realizado varias encuestas sobre el empleo del tiempo (Tabla 1). El primer antecedente se constituye en el año 1998 cuando se encuestó a mujeres de 14 años y más en la CABA por iniciativa del Sindicato de Amas de Casa de la República Argentina y el Consejo Nacional de la Mujer. Se trata de la Encuesta de Distribución del Uso del Tiempo, útil para conocer cómo ocupan las mujeres su tiempo según perfiles construidos en base a datos sociodemográficos (Rupnik y Colombo, s.f.). En el reporte de esta encuesta se explica que el monto producido por el trabajo no remunerado más el remunerado que realizan las mujeres es 1,57 veces más que el trabajo remunerado de los varones, y que las mujeres producen el $60 \%$ de los bienes y servicios que consume la sociedad. Destacan también que el trabajo doméstico no sólo carece de una remuneración sino también de días libres, feriados y retiro.

Luego, en el año 2001 se realizó la Encuesta de Condiciones de Vida (ECV) en la que se relevó información sobre el tiempo global dedicado a una lista de actividades domésticas. Este relevamiento tiene una representación de aproximadamente el $96 \%$ de la población urbana del país y, además de la ventaja de una mayor cobertura que la encuesta anterior, permite realizar un análisis integrado con datos sociodemográficos y socioeconómicos tanto para mujeres como para hombres.

En el año 2005 se recolectó, también en CABA, la Encuesta de Uso del Tiempo como un módulo adicional a la Encuesta Anual de Hogares. Esta encuesta, cuenta con las ventajas propias del instrumento utilizado, siendo la primera en el país que se implementa con el diario de actividades del día anterior. En este caso se realizaron registros en 48 bloques de 30 minutos para cada individuo encuestado (DGEyC de CABA, 2007). Bajo la misma metodología se realizó una encuesta en la Ciudad de Rosario en Santa Fe en el año 2010. 


\section{Tabla 1}

Características metodológicas de las encuestas de uso del tiempo en la Argentina

\begin{tabular}{|c|c|c|c|c|c|}
\hline Nombre & Año & $\begin{array}{l}\text { Cobertura } \\
\text { geográfica }\end{array}$ & Universo & Estrategia & Instrumento \\
\hline $\begin{array}{l}\text { Encuesta de Distribución del } \\
\text { Uso del Tiempo }\end{array}$ & 1998 & CABA & $\begin{array}{l}\text { Mujeres mayores a } 14 \\
\text { años inclusive. }\end{array}$ & $\begin{array}{l}\text { Encuesta } \\
\text { independiente }\end{array}$ & $\begin{array}{l}\text { Lista de } \\
\text { actividades }\end{array}$ \\
\hline $\begin{array}{l}\text { Encuesta de Condiciones de } \\
\text { Vida }\end{array}$ & 2001 & $\begin{array}{l}96 \% \text { de la } \\
\text { población } \\
\text { urbana } \\
\text { nacional. }\end{array}$ & $\begin{array}{l}\text { Hombres y mujeres } \\
\text { mayores a } 5 \text { años inclusive }\end{array}$ & Módulo & $\begin{array}{l}\text { Lista de } \\
\text { actividades }\end{array}$ \\
\hline Encuesta de Uso del Tiempo & 2005 & CABA & $\begin{array}{l}\text { Hombres y mujeres entre } \\
15 \text { y } 74 \text { años inclusive. }\end{array}$ & Módulo & $\begin{array}{l}\text { Diario de } \\
\text { actividades del día } \\
\text { anterior }\end{array}$ \\
\hline $\begin{array}{l}\text { Encuesta de Uso del Tiempo } \\
\text { y Voluntariado }\end{array}$ & 2010 & $\begin{array}{l}\text { Rosario, Santa } \\
\text { Fe }\end{array}$ & $\begin{array}{l}\text { Hombres y mujeres } \\
\text { mayores a } 15 \text { años } \\
\text { inclusive. }\end{array}$ & $\begin{array}{l}\text { Encuesta } \\
\text { independiente }\end{array}$ & $\begin{array}{l}\text { Diario de } \\
\text { actividades del día } \\
\text { anterior }\end{array}$ \\
\hline $\begin{array}{l}\text { Encuesta sobre Trabajo no } \\
\text { Remunerado y Uso del } \\
\text { Tiempo }\end{array}$ & 2013 & $\begin{array}{l}\text { Población } \\
\text { urbana nacional }\end{array}$ & $\begin{array}{l}\text { Hombres y mujeres } \\
\text { mayores a } 18 \text { años } \\
\text { inclusive. }\end{array}$ & Módulo & $\begin{array}{l}\text { Lista de } \\
\text { actividades }\end{array}$ \\
\hline
\end{tabular}

Nota: Elaboración propia

Finalmente, en el año 2013 el INDEC relevó la Encuesta sobre Trabajo no Remunerado y Uso del Tiempo como un módulo adicional a la Encuesta Anual de Hogares Urbanos (EAHU). Este trabajo utiliza datos de dicha encuesta. El cuestionario se aplicó a todas las personas mayores de 18 años seleccionadas en el muestreo de la EAHU. Según el diseño muestral, se cuenta con una cobertura en las estimaciones de la totalidad de la población urbana comprendida en ese rango etario.

Dicha encuesta consiste en un relevamiento sobre el trabajo doméstico no remunerado y el trabajo voluntario. En este documento se priorizan las actividades de trabajo no remunerado de cuidado ${ }^{4}$, por lo que las preguntas a considerar son:

Ayer,

1) ¿Cuánto tiempo le dedicó a: limpieza de casa, aseo y arreglo de ropa, preparar y cocinar alimentos, compras para el hogar, reparación y mantenimiento del hogar?

2) ¿Cuánto tiempo le dedicó al apoyo en tareas escolares a miembros del hogar?

3) ¿Cuánto tiempo le dedicó al cuidado de niños / enfermos o adultos mayores, miembros del hogar? (Incluye tiempos de traslado a actividades de cuidado).

La suma de horas que arrojan estas tres preguntas totaliza la cantidad de horas destinadas al trabajo no remunerado de cuidados.

\footnotetext{
${ }^{4}$ Según la definición del Fondo de Desarrollo de las Naciones Unidas para la Mujer de trabajo no remunerado de cuidado (UNIFEM, 2005).
} 


\section{Metodología}

\section{Medición de la pobreza LIMTIP}

Siguiendo la metodología LIMTIP medir pobreza en ingresos y tiempo implica construir dos líneas de pobreza: una que refleja el mínimo de ingreso necesario para acceder a una canasta básica de bienes y servicios producidos en el mercado, y otra que indica el mínimo de tiempo necesario para la producción doméstica. Con estos umbrales se construyen cuatro grupos de individuos: pobres por ingreso y por tiempo, no pobres por ingreso y pobres por tiempo, pobres por ingreso y no pobres por tiempo, y no pobres.

Zacharías et al. (2012) presentan el modelo que se utiliza en esta investigación. El modelo parte de una restricción de tiempo, se trata de una identidad de asignación de tiempo que establece que la cantidad total de horas semanales es igual a la suma del tiempo destinado a generar ingresos (trabajo remunerado o $T R$ ), a la producción doméstica (trabajo no remunerado o TNR), al cuidado personal y producción doméstica no sustituible $(C)$ y al tiempo libre o de ocio $(O)$. Esta restricción puede ser representada como:

(1) $168=T R_{i}+T N R_{i}+C_{i}+O_{i}$, donde $i$ : individuos en edad de trabajar.

A partir de esta identidad se deduce la ecuación (2) de déficit de tiempo individual. Para ello se mantiene la cantidad de horas de trabajo remunerado y se reemplaza el resto de las variables por sus umbrales de tiempo mínimo requerido por cada individuo $i$ en edad de trabajar $^{5}$ que viven en el hogar $j$. Así, $D_{i j}$ menor a cero implica que el individuo $i$ tiene déficit de tiempo, mientras que valores nulos o positivos implican disponibilidad de tiempo libre nulo o positivo.

(2) $D_{i j}=168-T R_{i j}-\alpha_{i j} t n r_{j}-c$

En el término correspondiente al trabajo no remunerado se agrega el parámetro $\alpha_{i j}$ que representa la disparidad en la división de tareas domésticas; se trata de la participación del individuo $i$ en el tiempo total que su hogar $j$ necesita de producción doméstica $\left(t n r_{j}\right)$. Dado que el tiempo requerido por el hogar para esa actividad varía según su

\footnotetext{
${ }^{5}$ Lamentablemente no se cuenta con datos de uso del tiempo de la población menor a 18 años, por lo que se desconoce su nivel de déficit individual y no es posible abordar temáticas como el trabajo infantil.
} 
composición demográfica, se construyen 12 grupos de referencia que surgen de combinar: 0, 1, 2 y 3 o más niños con 1, 2 o 3 adultos. Además, para determinar la cantidad de tiempo mínimo necesario de producción doméstica se promedia las horas utilizadas por hogares con al menos un adulto no empleado y con un ingreso cercano a la línea de pobreza monetaria. Se excluyen del grupo de referencia los hogares con todos sus adultos ocupados ya que se considera que esos hogares no serían capaces de gastar la cantidad suficiente de tiempo en producción doméstica al nivel de ingreso de la línea de pobreza tradicional (Zacharías et al., 2012).

Este procedimiento es análogo al que se realiza en la medición de pobreza monetaria. Allí, habitualmente, en base a un grupo de referencia se construye una canasta básica alimentaria. Como la estructura de consumo varía entre los hogares según su composición y su nivel de bienestar, se utiliza como referencia la estructura de la canasta de hogares cuyo consumo de alimentos satisface estrictamente, o supera levemente, los requerimientos nutricionales mínimos (DNEH, INDEC, 2003). De manera similar, la medición LIMTIP define grupos de referencia para determinar el consumo de tiempo en producción doméstica requerido para subsistir al nivel de pobreza por ingreso.

Por su parte, el umbral de tiempo mínimo de cuidado personal y producción doméstica no sustituible $(c)$ aparece como una constante, se supone igual para todos los individuos. Incluye actividades tales como dormir, comer, beber, actividades de higiene personal, etc., que hacen al cuidado personal, y también incorpora la idea de producción doméstica no sustituible que integra tareas domésticas que solo pueden ser realizadas por uno mismo; es la cantidad mínima de tiempo que los miembros del hogar necesitan destinar al manejo del hogar o a la interacción con otros miembros del hogar para reproducirlo como una unidad.

(3) $D_{j}=\sum_{i=1}^{n} \min \left(0, D_{i j}\right)$

Luego, siguiendo la ecuación (3) se agregan los déficits de los $n$ miembros adultos en cada hogar para obtener el nivel de deficiencia de tiempo del hogar. Cada miembro aportará al déficit del hogar el valor de su déficit si es deficitario, o cero si en cambio su nivel de déficit individual es nulo o positivo. Anulando el superávit que algún miembro del hogar pueda tener a la vez que otro sea deficitario, se evita que el excedente de tiempo del primero compense el déficit del segundo. Con eso, prescindimos del 
supuesto presente en medidas de pobreza de ingreso usuales, de que al interior del hogar la distribución del consumo es "justa" (acorde a las necesidades), y que un hogar pobre lo es porque no alcanza a cubrir en conjunto un nivel de consumo mínimo. Aquí, se toma la distribución observada y el hogar será deficitario si al menos uno de sus miembros lo es (Esquivel, 2014).

Se presenta el siguiente ejemplo para clarificar la razón por la que no se considera que excedentes de tiempo compensan déficits: pensemos en un hogar conformado por un matrimonio en el que ambos trabajan a jornada completa y en el que la mujer tiene déficit de tiempo porque es quien carga con gran parte de las tareas domésticas. Permitir que se compensen los déficits de algunos miembros con superávits de otros implicaría que el marido cambie su comportamiento de manera automática para aliviar el déficit de su esposa. Sin embargo, se entiende que ese cambio no es automático. De todas maneras, como se verá más adelante, hay otros medios que pueden aliviar ese déficit.

Si un hogar reviste la condición de deficitario supondremos que su déficit proviene de la insuficiencia de tiempo para alcanzar el nivel requerido de producción doméstica sustituible. Claramente hay otras actividades que pueden generar o profundizar esa condición, entre otras, el exceso de horas de trabajo remunerado. Sin embargo, en este trabajo se ha abordado la identificación de la pobreza en tiempo a partir de una corrección de las medidas de pobreza por ingreso. Como se explicó en secciones anteriores, se tiene en cuenta la necesidad de disponer de una persona que pueda trabajar a tiempo completo en el hogar, o la cantidad de horas necesarias para la producción doméstica. Por lo tanto, la carencia de tiempo y su posible alivio serán entendidos desde la escasez de tiempo para la producción doméstica sustituible.

La Figura 1 de este documento muestra que existe cierto grado de sustitución entre el tiempo y el nivel de ingreso. En esto términos, sería posible compensar el déficit de tiempo adquiriendo sustitutos provenientes del mercado, bastaría con cubrir el costo de reemplazo. Esta idea permite expresar a las variables temporales en términos monetarios, y construir una única línea de pobreza ajustada como se muestra en la expresión (4). La línea de pobreza monetaria ajustada está compuesta por la línea de pobreza tradicional $(\bar{y})$ y por un término que representa el costo de reemplazo de la producción doméstica. Ese costo se construye utilizando la condición de déficit de 
tiempo del hogar (que toma valores negativos o cero) y la unidad de costo de reemplazo, $p$.

(4) $y_{j}^{0}=\bar{y}-D_{j} p$

Veamos que si un hogar tiene déficit de tiempo, $D_{j}<0$, ese déficit de tiempo se transforma en términos monetarios a través de $p$, y ese hogar tendrá una línea de pobreza todavía más alta que aquella que se construye solo considerando la dimensión monetaria. Mientras mayor sea el nivel de déficit (en términos absolutos) mayor será el umbral de ingreso que deberá alcanzar ese hogar para escapar de la pobreza. Si el hogar no tiene escasez de tiempo, entonces su condición de pobreza estará determinada por la comparación entre su nivel de ingreso y la línea de pobreza tradicional.

Los resultados que se suelen presentar con esta metodología son cuatro grupos según la condición de pobreza: pobres por ingreso y tiempo, no pobre por ingreso y pobre en tiempo, pobre por ingreso y no pobre en tiempo y no pobre en ninguna dimensión. De esta manera se hace visible la pobreza oculta, es decir, aquella que padecen los individuos que superando la línea de pobreza monetaria tradicional no son considerados pobres por las estadísticas oficiales, pero que sin embargo, pueden estar soportando una situación de explotación dada la gran cantidad de horas que dedican a la generación de valor económico (es decir, al trabajo remunerado y no remunerado).

\section{Aplicación empírica}

Como se explicó en la sección anterior, estimar el déficit de tiempo de una persona requiere información sobre cómo esa persona distribuye su tiempo en diferentes actividades. Para facilitar la estimación se clasifica a las actividades en cuidado personal, trabajo doméstico y trabajo remunerado. Sustituyendo los valores conocidos de la cantidad de horas destinadas a cada actividad en la ecuación (2) se obtiene el déficit de tiempo individual para cada persona de 18 años y más. Sin embargo, el módulo sobre uso del tiempo vinculado a la EAHU 2013 no cuenta con información suficiente como para estimar todos los umbrales ${ }^{6}$. Es por eso que se optó por utilizar umbrales construidos en trabajos anteriores basados en la encuesta de uso del tiempo de CABA 2005 (Zacharías et al., s.f.).

\footnotetext{
${ }^{6}$ Como la encuesta utilizada tampoco cuenta con datos sobre el uso del tiempo para menores de 18 años no se ha incorporado en el análisis a aquellos hogares sin adultos, pues no se podría precisar su situación de déficit en tiempo. Por esta causa se ha perdido información, por ejemplo, relativa a hogares con parejas adolescentes.
} 
Utilizar dichos umbrales implica suponer que en el año 2013 los argentinos necesitaban la misma cantidad de horas para el cuidado personal y la producción doméstica no sustituible $(c)$, la misma cantidad de tiempo para realizar la producción doméstica sustituible según la composición del hogar (tnr), y la misma cantidad de tiempo para trasladarse desde y hacia el trabajo que en 2005 en CABA. Así, como indica la Tabla 2, cada individuo debería disponer de 94 horas semanales para el cuidado personal, 84 minutos para trasladarse a un trabajo de tiempo medio y 3,8 horas para hacerlo a uno de tiempo completo.

Tabla 2

Horas de producción sustituible por semana según composición del hogar

\begin{tabular}{ccccc}
\hline \multicolumn{5}{c}{ Horas de producción doméstica } \\
\hline \multirow{5}{*}{ Adultos } & $\mathbf{0}$ & $\mathbf{1}$ & $\mathbf{2}$ & $\mathbf{3}$ o más \\
\cline { 2 - 5 } Niños \\
$\mathbf{1}$ & 18 & 45 & 64 & 76 \\
$\mathbf{2}$ & 40 & 63 & 83 & 94 \\
$\mathbf{3}$ o más & 95 & 118 & 137 & 148 \\
\hline \multicolumn{6}{c}{} \\
Cuidado personal & & & Horas \\
Traslado al trabajo (medio tiempo) & 1,4 \\
Traslado al trabajo (tiempo completo) & 3,8 \\
\hline
\end{tabular}

Fuente: Tomado de Zacharías et al. (Sin fecha)

También se mantendrá la misma cantidad de horas mínimas requeridas para las tareas domésticas según la cantidad de miembros del hogar; por ejemplo, un hogar compuesto por 2 adultos y 2 niños necesita 83 horas semanales para reproducirse (Tabla 2). Como se dijo, utilizar los mismos umbrales supone que las necesidades mínimas de tiempo en tareas domésticas de los hogares que se encuentran alrededor de la línea de pobreza no han variado de manera sustancial entre 2005 y 2013. Dicho supuesto no resulta absurdo si consideramos que el tiempo destinado a las tareas domésticas está determinado por la productividad, y que puede mejorar (ser menor) gracias a avances tecnológicos que aporten novedosos electrodomésticos, a innovaciones en la industria alimenticia que provean alimentos preparados o semi-preparados, o a un mayor acceso a las comunicaciones, por ejemplo para realizar trámites vía internet en lugar de concurrir a una oficina y esperar un turno. Estos factores determinantes de la producción doméstica 
no han cambiado radicalmente en el periodo 2005-2013, al menos no para la población que se encuentra alrededor de la línea de pobreza y que constituye el grupo de referencia.

\section{Tabla 3}

Test de diferencia de medias en horas de trabajo por tipo de hogar según región, 2do. trimestre 2005 y 2 do. trimestre 2013

\begin{tabular}{|c|c|c|c|c|c|c|c|}
\hline \multirow{2}{*}{\multicolumn{2}{|c|}{ Tipo de hogar }} & \multicolumn{6}{|c|}{$\begin{array}{r}\text { Valor } P \text { [Ho: media } 2005=\text { media } 2013 ; \\
\text { Ha: } \text { media } 2005 \neq \text { media } 2013]\end{array}$} \\
\hline & & $\begin{array}{l}\text { CABA y } \\
\text { GBA }\end{array}$ & NOA & NEA & Cuyo & Pampeana & Patagónica \\
\hline \multirow{4}{*}{1 adulto } & 0 niños & 0,751 & 0,169 & 0,420 & 0,435 & 0,073 & 0.452 \\
\hline & 1 niño & 0,990 & 0,672 & 0,178 & 0,936 & 0,645 & 0.012 \\
\hline & 2 niños & 0,974 & 0,949 & 0,639 & 0,539 & 0,739 & 0.291 \\
\hline & 3 o más & 0,648 & 0,019 & 0,216 & 0,058 & 0,006 & 0.003 \\
\hline \multirow{4}{*}{2 adultos } & 0 niños & 0,941 & 0,030 & 0,398 & 0,010 & 0,000 & 0.310 \\
\hline & 1 niño & 0,688 & 0,096 & 0,984 & 0,588 & 0,637 & 0.029 \\
\hline & 2 niños & 0,966 & 0,107 & 0,003 & 0,022 & 0,067 & 0.264 \\
\hline & 3 o más & 0,673 & 0,025 & 0,174 & 0,532 & 0,192 & 0.091 \\
\hline \multirow{4}{*}{$\begin{array}{l}3 \text { adultos o } \\
\text { más }\end{array}$} & 0 niños & 0,666 & 0,308 & 0,886 & 0,465 & 0,011 & 0.561 \\
\hline & 1 niño & 0,879 & 0,174 & 0,653 & 0,076 & 0,050 & 0.009 \\
\hline & 2 niños & 0,660 & 0,284 & 0,787 & 0,203 & 0,000 & 0.674 \\
\hline & 3 o más & 0,731 & 0,028 & 0,183 & 0,447 & 0,019 & 0.000 \\
\hline \multicolumn{2}{|c|}{$\begin{array}{l}\text { Participación en la } \\
\text { población total }\end{array}$} & 0,54 & 0,10 & 0,05 & 0,06 & 0,23 & 0,02 \\
\hline \multicolumn{2}{|c|}{$\begin{array}{l}\text { Porcentaje de } \\
\text { personas cuyo tipo de } \\
\text { hogar rechaza la Ho } \\
\text { con un } 95 \% \text { de nivel } \\
\text { de confianza. }\end{array}$} & 0,00 & 4,34 & 0,47 & 1,18 & 13,88 & 0,81 \\
\hline
\end{tabular}

Fuente: Elaboración propia a partir de datos de la Encuesta Permanente de Hogares (EPH), años 2005 y 2013.

A modo de prueba de robustez de la escasa variación en la distribución del tiempo, se presenta en la Tabla 3 un test de diferencia de medias entre las horas de trabajo en 2005 y en 2013. Las diferencias se tomaron entre los promedios de cada tipo de hogar según regiones y teniendo en cuenta solamente las personas con horas de trabajo positivas (no nulas). Allí se puede observar que en la mayoría de los tipos de hogares no es posible rechazar la hipótesis nula de igualdad en los promedios de horas trabajadas por adultos de cada año. El porcentaje de personas que viven en hogares y regiones donde se 
rechaza la hipótesis nula con un $99 \%$ de confianza asciende al 6,6\%, y con un $95 \%$ de confianza, al 20,7\% y la mayoría corresponde a la región Pampeana.

En síntesis, siendo exigentes con el nivel de significancia del test, el 93,4\% de la población vive en hogares donde la cantidad de horas trabajadas por los adultos, en promedio, no ha variado sustancialmente en el período 2005-2013. Recordemos que el déficit de tiempo se construye a partir de las actividades trabajo remunerado, trabajo no remunerado y cuidado personal (ecuación 2). Como se ha fijado las horas de cuidado personal iguales a las necesarias en 2005 (ya que la encuesta de 2013 no contiene datos sobre cuidado personal pero se presume cierta estabilidad debido a que se trata del uso de tiempo en actividades biológicas necesarias y con cierta permanencia) y se ha comprobado escasa variación en el promedio de horas de trabajo remunerado entonces se supone adecuado suponer que la cantidad de horas de trabajo no remunerado - como complemento de esos componentes en la construcción del déficit de tiempo - también han sufrido, en promedio, variaciones mínimas (aunque no se cuenta con los elementos necesarios para evaluar esta variable).

Adicionalmente se realiza el mismo test para evaluar diferencias entre el promedio de horas de trabajo en la Ciudad de Buenos Aires y el resto del país ${ }^{7}$, puesto que se pretende utilizar los umbrales estimados para CABA en la medición de la pobreza LIMTIP del país. El valor P obtenido en el test de diferencias de medias es 0.643 , por lo tanto no es posible rechazar la hipótesis nula de igualdad de medias. Con estas pruebas se pretende respaldar el supuesto de escasa variación en la distribución del tiempo entre las diferentes actividades durante el periodo 2005-2013 y también la pertinencia de utilizar umbrales de CABA para estimar la pobreza en todo el país.

Por otra parte, según indica la ecuación (4) del modelo es necesario construir una línea de pobreza monetaria. Para identificar a los individuos en condición de pobreza monetaria se compara el ingreso total familiar con el valor de una canasta básica total cuyo ingreso debiera alcanzar para estar fuera de la pobreza.

Para obtener el valor de la canasta se utiliza el índice de precios al consumidor de la ciudad de $\operatorname{Salta}^{8}$ (puesto que no se cuenta con un índice nacional oficial para el año

\footnotetext{
${ }^{7}$ En este caso no se procede comparando tipos de hogares de CABA contra tipos de hogares del resto del país ya que los perfiles de Ciudad de Buenos Aires cuentan con muy pocas observaciones como para ser representativos.

${ }^{8}$ El relevamiento y estimación del índice en esta jurisdicción se mantuvo al margen de la intervención del INDEC. Se puede consultar una serie histórica de este índice en:
} 
2013) y la noción de adulto equivalente. Así, el valor de la canasta básica total para el adulto equivalente utilizado en cada región es: GBA \$1185,8; NOA \$966,7: NEA \$1003,0; Cuyo \$1133,8; Pampeana \$11862 y Patagónica \$1385,3.

\section{Análisis condicional}

Para el análisis de los determinantes o factores asociados a la condición de pobreza LIMTIP se aplica un modelo logit acorde a la variable cuyo comportamiento se pretende explicar. La variable dependiente es $y$, que toma valor uno si el individuo se identifica como pobre LIMTIP y cero si está fuera de la pobreza.

Los resultados de $y_{i}$ ocurren con una probabilidad $\pi_{i}$ que es una probabilidad condicional a las variables explicativas consideradas:

$\pi_{i} \equiv \operatorname{Pr}\left(y_{i}\right) \equiv \operatorname{Pr}\left(y_{i} \mid x\right)$

donde $x$ es el conjunto de variables explicativas, que en este contexto son los determinantes profundos de la pobreza LIMTIP.

Para modelar la probabilidad de que un individuo sea pobre LIMTIP se propone aquí un modelo con variable dependiente binaria, que puede ser escrito de la manera siguiente:

$\operatorname{Pr}(y=1 \mid x)=G(x \beta)$

donde $\mathrm{G}$ es una función que toma valores cero y uno, como se explicó antes. Para asegurar de que la media condicional se sitúe entre cero y uno, se propone para $\mathrm{G}$ una forma funcional no lineal que sigue una función de distribución normal:

$G(z)=\int_{-\infty}^{Z} \frac{1}{(2 \pi)^{1 / 2}} e^{s^{2} / 2} d s+\varepsilon_{i}$

Esta es la función de distribución acumulada de una variable aleatoria normal estandarizada. La función $G$ es creciente en $z=0, G(z) \rightarrow 0$ cuando $z \rightarrow-\infty$ y $G(z) \rightarrow 1$ cuando $z \rightarrow \infty$.

La interpretación de los coeficientes estimados puede entenderse mejor apelando al concepto de Odds ratios (OR) o cocientes de probabilidades. Dicho concepto puede ser expresado analíticamente como:

$O R=\frac{\operatorname{Pr}(y=1)}{1-\operatorname{Pr}(y=1)}=\exp (x \beta)$

http:/estadisticas.salta.gov.ar/web/archivos/anuarios/anuario2014-2015.pdf (Última fecha de consulta: 31 de octubre de 2016). 
Aplicando logaritmos en ambos miembros de la expresión anterior:

$\operatorname{logit}[\operatorname{Pr}(y=1)]=\ln \left[\frac{\operatorname{Pr}[(y=1)}{1-\operatorname{Pr}[(y=1)}\right]=x \beta$

Por lo tanto, el coeficiente $\beta$ indica el cambio en el logit (o en el logaritmo natural del cociente de probabilidades) debido a un aumento en una unidad en la variable correspondiente, mientras se mantienen constantes las demás variables explicativas.

\section{Resultados}

\section{Medición de la pobreza LIMTIP}

La pobreza monetaria en la Argentina en 2013 asciende a 17,1\% de la población urbana. Como se puede apreciar en el Gráfico 1, la pobreza LIMTIP en ese mismo año es 19,6\%. La diferencia arroja como resultado 2,5 puntos porcentuales (p.p.) de pobres ocultos, alrededor de 1 millón de personas con ingresos superiores al umbral de pobreza monetaria pero inferiores a la línea de pobreza según la medición LIMTIP. A nivel regional la brecha es mayor: NOA 4 p.p. y NEA 4,5 p.p. Aparentemente, en estas regiones hay una acumulación mayor de población vulnerable (aquella fuera de la pobreza pero con un ingreso muy cercano al umbral, por lo que con altas probabilidades de caer en la pobreza).

Gráfico 1

Pobreza monetaria y pobreza LIMTIP. Argentina y regiones, 2013

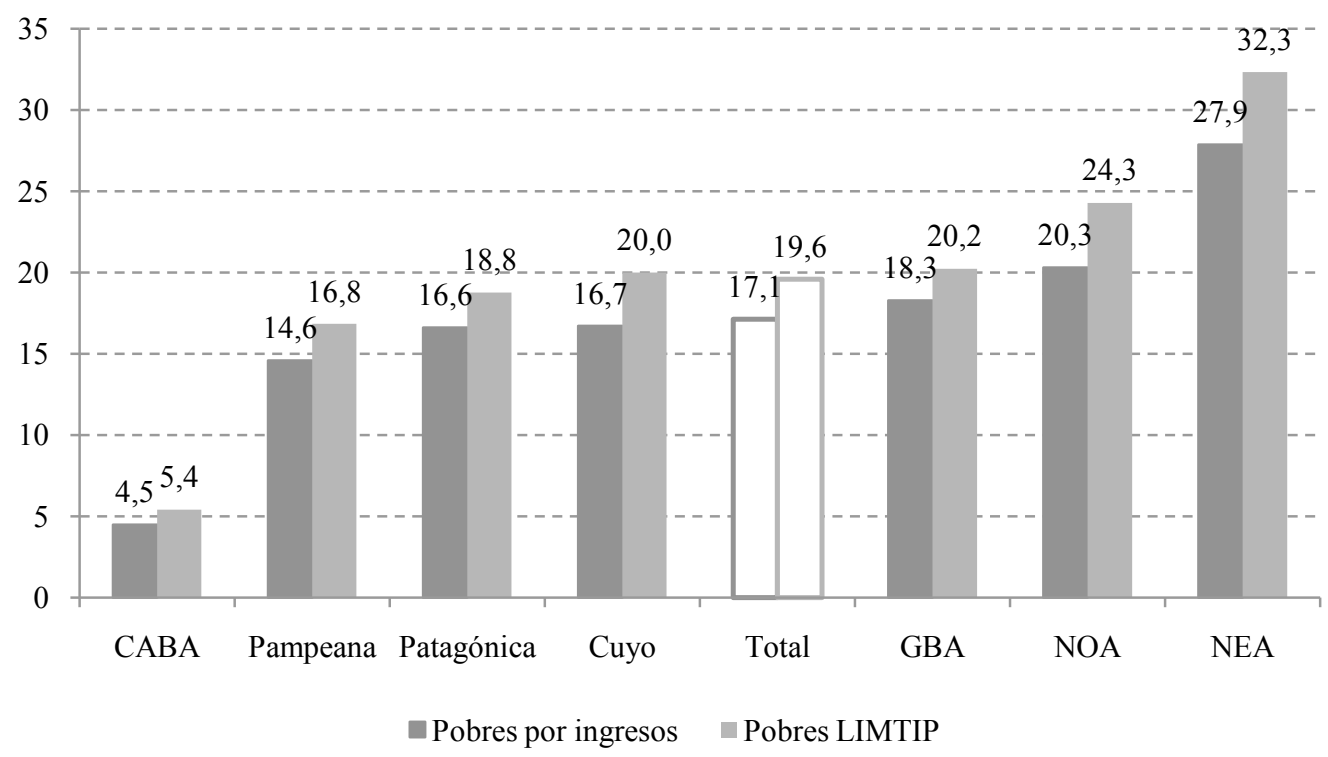

Fuente: Elaboración propia a partir de datos de la Encuesta sobre Trabajo no Remunerado y Uso del Tiempo, año 2013. 
Quienes están sumidos en la condición de pobreza monetaria no logran cubrir la canasta básica total con sus ingresos, ni disponen del tiempo suficiente para realizar las tareas necesarias de reproducción del hogar. Los pobres ocultos alcanzan la canasta, pero no cuentan con los ingresos suficientes como para sustituir su déficit de tiempo, ni margen de tiempo como para generar mayores ingresos. Aunque el nivel de bienestar de los pobres ocultos se ve debilitado por el déficit de ingreso ajustado en función del tiempo, no son considerados pobres bajo las medidas oficiales, y por lo tanto las políticas de reducción de pobreza no los alcanzan. Las regiones más afectadas por la pobreza monetaria son también las de mayor incidencia de pobres ocultos y por lo tanto de pobreza LIMTIP. Esta evidencia sugiere que la magnitud del efecto del déficit de tiempo en la generación de pobreza LIMTIP en las regiones menos pobres por ingresos no sea tal que iguale la mayor insuficiencia de ingresos en otras regiones.

En la Tabla 4 se presenta la condición de pobreza según grupos de población. Allí se observa que el grupo de menores de 18 años es el más afectado; más de un cuarto de la población está en condición de pobreza. Este grupo poblacional es también el más afectado por la pobreza LIMTIP ya que aproximadamente 3 de cada 10 niños viven en un hogar donde el ingreso familiar ajustado por el déficit de tiempo de los adultos es insuficiente. Además, la pobreza entre los niños más que duplica la pobreza entre los adultos. Posiblemente se deba a que los hogares pobres, en general, son hogares con mayor número de menores y esa carga incide tanto en términos de ingreso como en demanda de tiempo, por lo que las dos dimensiones consideradas en la medición se potencian.

\section{Tabla 4}

Incidencia de la pobreza monetaria y la pobreza LIMTIP para grupos de población seleccionados. Argentina, 2013

\begin{tabular}{lcc}
\hline & $\begin{array}{c}\text { Pobreza } \\
\text { monetaria }\end{array}$ & $\begin{array}{c}\text { Pobre } \\
\text { LIMTIP }\end{array}$ \\
\hline Hombres (18 años y más) & 12,56 & 14,43 \\
Mujeres (18 años y más) & 12,79 & 14,68 \\
Niños y adolescentes & 27,38 & 31,26 \\
\hline
\end{tabular}

Fuente: Elaboración propia a partir de datos de la Encuesta sobre Trabajo no Remunerado y Uso del Tiempo, año 2013.

De la Tabla 4 también surge que la diferencia tanto en pobreza monetaria como en pobreza LIMTIP entre hombres y mujeres no es significativa. Por lo tanto la magnitud de la población oculta es igual en ambos géneros. 
El patrón de mayor presencia de pobreza en la población infantil se repite en todas las regiones. Según se puede observar en la Tabla 5, los niños y adolescentes aportan en cada región alrededor del $50 \%$ de la pobreza LIMTIP mientras sólo representan aproximadamente un tercio de la población. Más aun, en algunas regiones la contribución a la pobreza LIMTIP más que duplica el aporte a la población como en el caso de CABA, donde la participación de la niñez y adolescencia entre los pobres LIMTIP es $56 \%$ contra $23,5 \%$ de su participación en la población.

Tabla 5

Contribución de cada grupo poblacional en la pobreza LIMTIP y en la población total de la región, y de cada región a la pobreza LIMTIP nacional y en la población total del país. Argentina, 2013

\begin{tabular}{|c|c|c|c|c|c|c|}
\hline & & $\begin{array}{l}\text { Hombres } \\
(+18 \text { años })\end{array}$ & $\begin{array}{c}\text { Mujeres } \\
(+18 \text { años })\end{array}$ & $\begin{array}{c}\text { Niños y } \\
\text { adolescentes }\end{array}$ & Total & $\begin{array}{c}\text { Región en el } \\
\text { total país }\end{array}$ \\
\hline \multirow{2}{*}{ CABA } & Pobreza LIMTIP & 17,77 & 26,23 & 56,00 & 100 & 2,16 \\
\hline & Población & 35,05 & 41,47 & 23,49 & 100 & 7,81 \\
\hline \multirow{2}{*}{ GBA } & Pobreza LIMTIP & 23,38 & 27,35 & 49,26 & 100 & 37,80 \\
\hline & Población & 32,62 & 36,62 & 30,76 & 100 & 36,62 \\
\hline \multirow{2}{*}{ NOA } & Pobreza LIMTIP & 25,65 & 27,51 & 46,84 & 100 & 13,76 \\
\hline & Población & 31,51 & 34,76 & 33,73 & 100 & 11,10 \\
\hline \multirow{2}{*}{ NEA } & Pobreza LIMTIP & 23,03 & 26,48 & 50,49 & 100 & 13,54 \\
\hline & Población & 30,46 & 34,22 & 35,32 & 100 & 8,21 \\
\hline \multirow{2}{*}{ Cuyo } & Pobreza LIMTIP & 24,83 & 28,59 & 46,58 & 100 & 6,74 \\
\hline & Población & 32,46 & 36,79 & 30,76 & 100 & 6,60 \\
\hline \multirow{2}{*}{ Pampeana } & Pobreza LIMTIP & 25,93 & 28,15 & 45,91 & 100 & 20,86 \\
\hline & Población & 34,45 & 38,15 & 27,41 & 100 & 24,28 \\
\hline \multirow{2}{*}{ Patagónica } & Pobreza LIMTIP & 25,19 & 29,67 & 45,14 & 100 & 5,14 \\
\hline & Población & 32,72 & 34,48 & 32,80 & 100 & 5,38 \\
\hline \multirow{2}{*}{ Total } & & & & & & 100 \\
\hline & & & & & & 100 \\
\hline
\end{tabular}

Fuente: Elaboración propia a partir de datos de la Encuesta sobre Trabajo no Remunerado y Uso del Tiempo, año 2013.

La evidencia muestra que la incidencia de la pobreza LIMTIP no difiere entre géneros (Tabla 3), no obstante como se observa en la Tabla 5 el segundo grupo que contribuye más a la pobreza LIMTIP es el de las mujeres mayores de 18 años, después del grupo de niños y adolescentes. Este indicador de contribución a la pobreza no solo tiene bajo consideración la incidencia sino también la participación del grupo en la población total otorgando una idea de la magnitud del aporte que hace éste a la pobreza LIMTIP. En algunas regiones la magnitud de la contribución de hombres y mujeres no difiere de manera significativa aunque en otras la diferencia ronda los 10 puntos, como es el caso de CABA. 
Además de las desigualdades intrarregionales que afectan más a la población menor de edad, también existen diferencias entre regiones. Así, las regiones que conforman el norte del país (NOA y NEA) contribuyen a la pobreza muy por encima de su participación en la población total, principalmente el NEA. No obstante no son las regiones de mayor contribución a la pobreza total dada su baja participación en la población. GBA y la región Pampeana, las regiones más populosas, concentran el $60 \%$ de la pobreza LIMTIP.

\section{Gráfico 3}

Pobreza LIMTIP por sexo del jefe según composición del hogar. Argentina, 2013

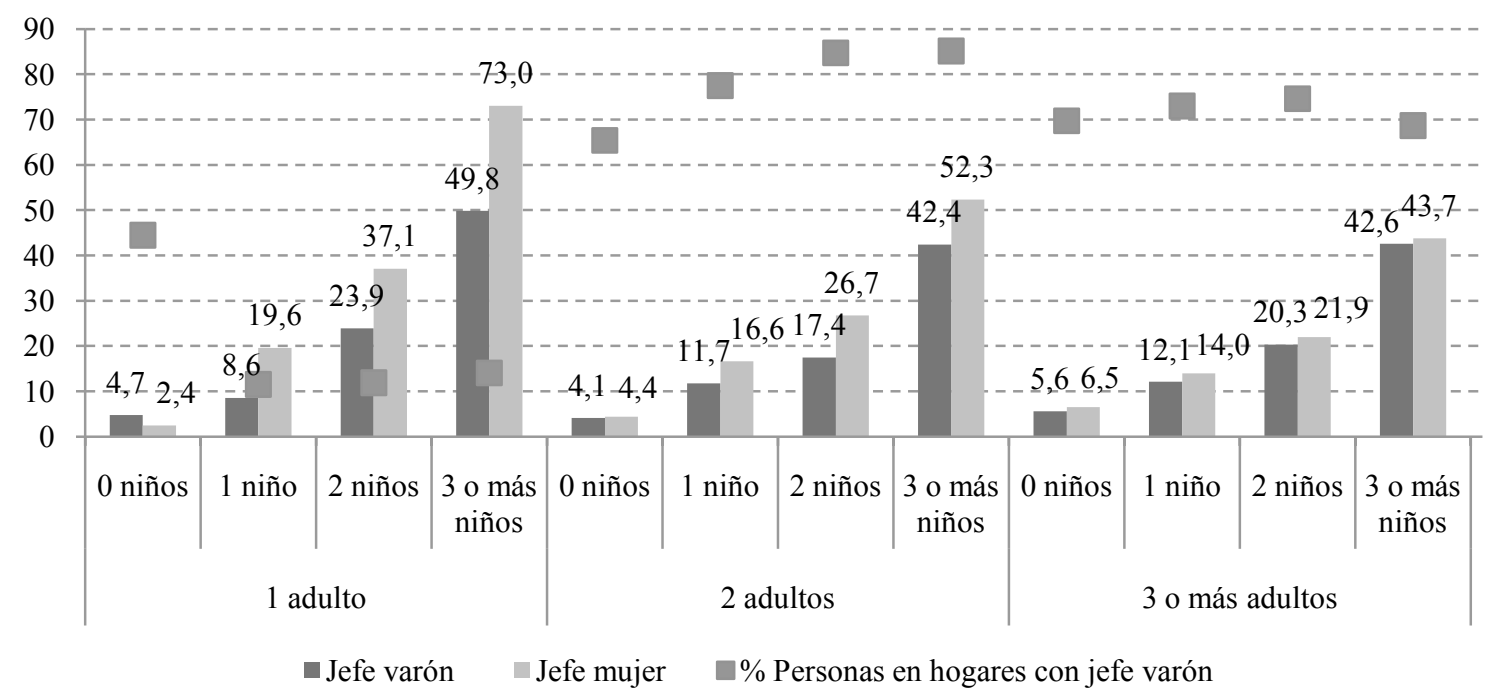

Fuente: Elaboración propia a partir de datos de la Encuesta sobre Trabajo no Remunerado y Uso del Tiempo, año 2013.

Nota: La diferencia entre la incidencia de pobreza LIMTIP para personas con jefatura femenina y masculina resulta estadísticamente significativa al $1 \%$ en cada tipo de hogar.

Otro factor asociado a la condición de pobreza LIMTIP es la composición del hogar, por eso se muestra el Gráfico 3 en relación a la estructura del hogar y el sexo del jefe. Allí surge, primero, que la mayoría de las personas que vive en hogares con un adulto y niños a cargo, lo hace bajo jefatura femenina. Entre los hogares monoparentales con niños la participación de la jefatura femenina ronda el 90\%. Por otra parte, los hogares unipersonales se distribuyen más o menos igual entre jefes varones y mujeres, y este tipo de hogar es uno de los dos únicos tipos en los que la incidencia de pobreza LIMTIP es mayor entre las personas cuyo jefe es varón, además de aquel compuesto por 3 o más adultos y 2 niños; el resto de los hogares siempre se encuentra lo contrario (mayor incidencia en hogares con jefatura femenina).

Puntualmente, se observa que la incidencia de la pobreza LIMTIP es mayor a medida que los hogares tienen más niños. Así, el porcentaje más alto se encuentra entre las 
personas en hogares con 3 o más niños y ronda el 50\%, duplicando la incidencia de aquellos hogares con solo 2 niños. Los hogares más agobiados por la pobreza LIMTIP son aquellos habitados por una mujer y 3 o más niños: sólo un cuarto de ese tipo de hogares logra escapar de la pobreza.

Otro factor relevante en la distribución del tiempo es el tipo de ocupación, y en alguna medida la pobreza LIMTIP puede ser considerada un indicador de la calidad del trabajo tanto por reflejar la insuficiencia de ingresos como el exceso de jornadas laborales de los empleados. Con todo, se puede observar en el Gráfico 4 que la incidencia de la pobreza LIMTIP es alta entre los desocupados, trabajadores familiares sin remuneración, asalariados no registrados y cuentapropistas no profesionales. En otras palabras, es alta entre los trabajadores más desaventajados.

\section{Gráfico 4}

Pobreza LIMTIP por sexo según estado ocupacional. Argentina, 2013

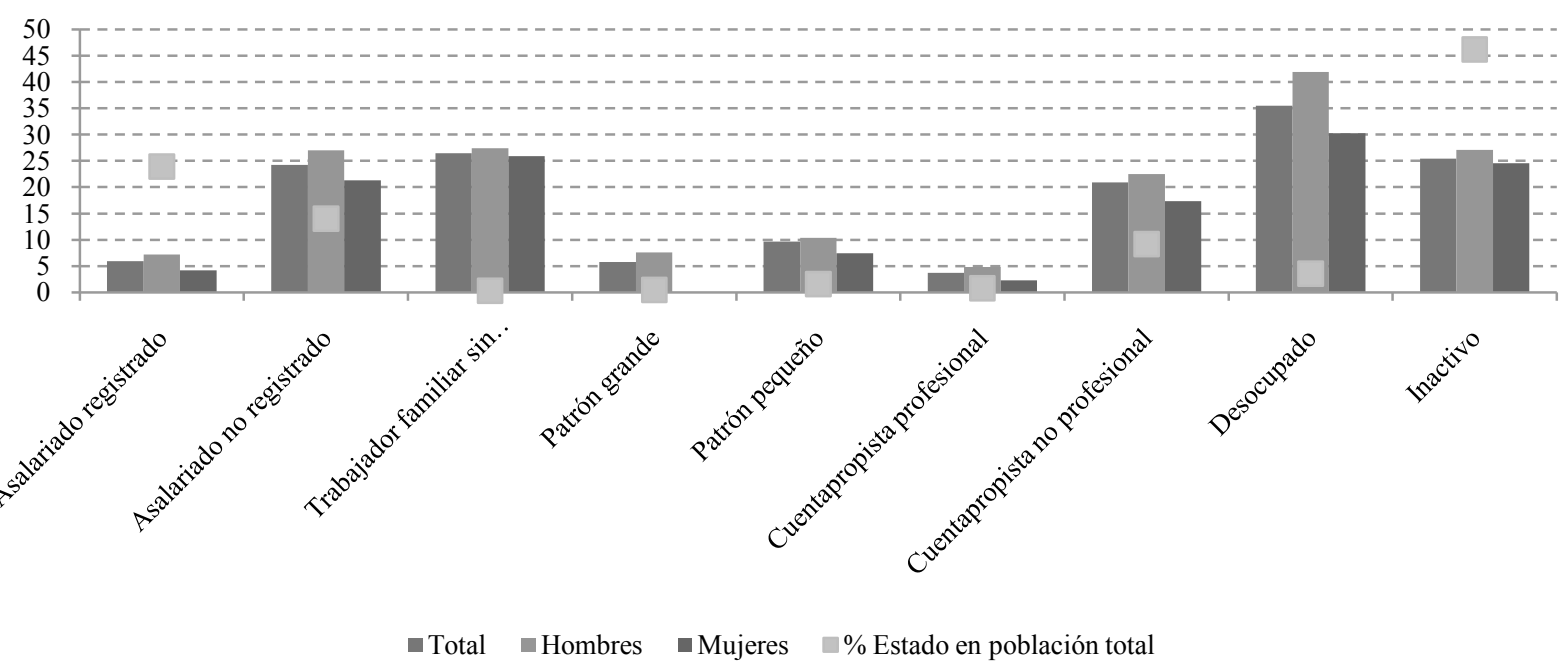

Fuente: Elaboración propia a partir de datos de la Encuesta sobre Trabajo no Remunerado y Uso del Tiempo, año 2013.

La incidencia de pobreza LIMTIP entre los asalariados no registrados es aproximadamente 4 veces mayor que la de los registrados. Por su parte, la tasa de pobreza LIMTIP entre los trabajadores familiares es alta, pero la proporción de este tipo de trabajadores en el total es ínfima ( $0,3 \%)$. Entre los desocupados y los inactivos, como no prestan horas de trabajo al mercado, seguramente el alto nivel de pobreza LIMTIP está determinado por las importantes cargas horarias en las tareas domésticas sumado a la pobreza por ingreso. La caracterización de la pobreza LIMTIP en relación al estado ocupacional arroja valores menores entre las mujeres que entre los hombres. 


\section{Gráfico 5 \\ Composición de la pobreza LIMTIP según condición de ocupación. Argentina, 2013}

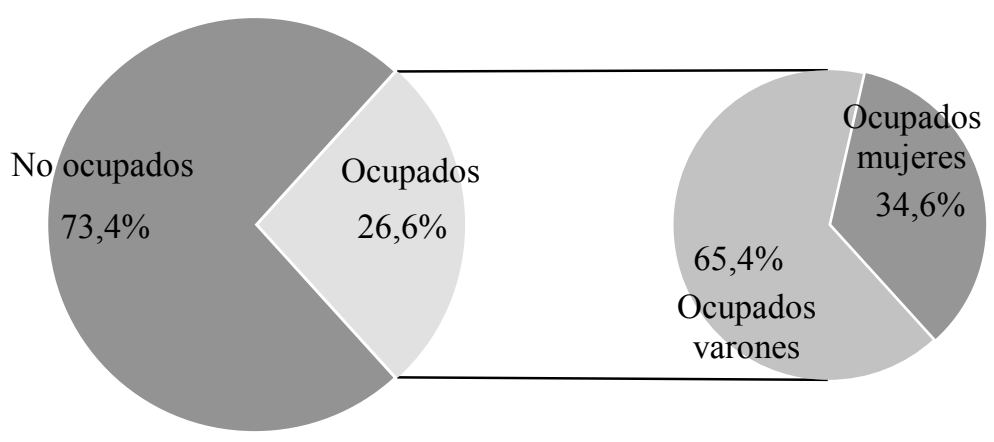

Fuente: Elaboración propia a partir de datos de la Encuesta sobre Trabajo no Remunerado y Uso del Tiempo, año 2013.

Por último, se presenta el Gráfico 5 con intención de mostrar que la mera creación de empleo no resuelve el problema de la pobreza. El gráfico de torta de la izquierda muestra cómo se reparte la población pobre LIMTIP entre ocupados y no ocupados. El de la derecha, divide el total de ocupados y pobres LIMTIP por género. Allí se puede observar que el 73,4\% de la población pobre LIMTIP no está ocupada, son inactivos o desocupados, mientras que el restante $26,6 \%$ sí lo está. Estos resultados sugieren que una persona puede tener un empleo pero a la vez revestir la condición de pobre por escasez de ingreso y/o de tiempo. En este caso un cuarto de la población pobre LIMTIP están ocupados, aunque probablemente el ingreso laboral de este grupo no es suficiente para alcanzar un nivel de vida aceptable o sus jornadas de trabajo son demasiado extensas, situaciones que los mantienen en la pobreza.

\section{Análisis condicional}

Tras haber realizado un análisis descriptivo del vínculo entre la condición de pobreza LIMTIP y algunas variables que pueden llegar a explicar la mayor o menor probabilidad de caer en ella, se agrega un análisis econométrico que permite obtener una primera aproximación de posibles determinantes de la pobreza, además de identificar la significancia estadística. Se muestra en la Tabla 6 un modelo de regresión logística para explicar la condición de pobreza LIMTIP cuyos coeficientes están en la columna (1) y los valores de la razón de probabilidad en la (2). 
Tabla 6

Modelo de regresión logística y Odds ratio de la condición de pobreza LIMTIP

\begin{tabular}{|c|c|c|}
\hline & $\begin{array}{c}(1) \\
\text { Pobre LIMTIP }\end{array}$ & $\begin{array}{c}(2) \\
\text { Razón de } \\
\text { probabilidad }\end{array}$ \\
\hline Años de educación del jefe de hogar & $\begin{array}{l}-0.105 * * * \\
(0.005)\end{array}$ & $\begin{array}{l}0.900 * * * \\
(0.005)\end{array}$ \\
\hline Jefe asalariado informal o cuentapropista no profesional & $\begin{array}{l}1.496^{* * * *} \\
(0.047)\end{array}$ & $\begin{array}{l}4.465^{* * *} \\
(0.211)\end{array}$ \\
\hline Jefe ocupado (resto) & $\begin{array}{l}0.751 * * * \\
(0.086)\end{array}$ & $\begin{array}{l}2.120^{* * *} \\
(0.183)\end{array}$ \\
\hline Jefe inactivo & $\begin{array}{l}1.586^{* * *} \\
(0.066)\end{array}$ & $\begin{array}{l}4.882^{* * *} \\
(0.323)\end{array}$ \\
\hline Jefe desocupado & $\begin{array}{l}2.565^{* * *} \\
(0.103)\end{array}$ & $\begin{array}{l}13.007 * * * \\
(1.335)\end{array}$ \\
\hline Jefe varón & $\begin{array}{c}0.077 \\
(0.048)\end{array}$ & $\begin{array}{l}1.079 \\
(0.052)\end{array}$ \\
\hline Cantidad de niñas menores de 5 años & $\begin{array}{l}0.563^{* * *} \\
(0.043)\end{array}$ & $\begin{array}{l}1.755^{* * *} \\
(0.076)\end{array}$ \\
\hline Cantidad de niños menores de 5 años & $\begin{array}{l}0.382 * * * \\
(0.032)\end{array}$ & $\begin{array}{l}1.465^{* * *} \\
(0.047)\end{array}$ \\
\hline Cantidad de mujeres entre 5 y 17 años & $\begin{array}{l}0.543^{* * *} \\
(0.026)\end{array}$ & $\begin{array}{l}1.721^{* * *} \\
(0.045)\end{array}$ \\
\hline Cantidad de varones entre 5 y 17 años & $\begin{array}{l}0.662^{* * *} \\
(0.022)\end{array}$ & $\begin{array}{l}1.939 * * * \\
(0.043)\end{array}$ \\
\hline Cantidad de mujeres entre 18 y 64 años & $\begin{array}{l}-0.182 * * * \\
(0.026)\end{array}$ & $\begin{array}{l}0.834 * * * \\
(0.021)\end{array}$ \\
\hline Cantidad de varones entre 18 y 64 años & $\begin{array}{l}-0.222 * * * \\
(0.027)\end{array}$ & $\begin{array}{l}0.800^{* * *} \\
(0.022)\end{array}$ \\
\hline Cantidad de mujeres entre 65 y 79 años & $\begin{array}{l}-2.116^{* * * *} \\
(0.124)\end{array}$ & $\begin{array}{l}0.121^{* * *} \\
(0.165)\end{array}$ \\
\hline Cantidad de varones entre 65 y 79 años & $\begin{array}{l}-0.658^{* * * *} \\
(0.151)\end{array}$ & $\begin{array}{l}0.518^{* * *} \\
(0.078)\end{array}$ \\
\hline Cantidad de mujeres de 80 años y más & $\begin{array}{l}-2.116^{* * *} \\
(0.137)\end{array}$ & $\begin{array}{l}0.121^{* * *} \\
(0.017)\end{array}$ \\
\hline Cantidad de varones de 80 años y más & $\begin{array}{l}-1.406^{* * * *} \\
(0.162)\end{array}$ & $\begin{array}{l}0.246^{* * *} \\
(0.040)\end{array}$ \\
\hline NOA & $\begin{array}{l}0.136^{* * *} \\
(0.052)\end{array}$ & $\begin{array}{l}1.146^{* * *} \\
(0.060)\end{array}$ \\
\hline NEA & $\begin{array}{l}0.446^{* * *} \\
(0.054)\end{array}$ & $\begin{array}{l}1.561^{* * *} \\
(0.085)\end{array}$ \\
\hline Cuyo & $\begin{array}{l}0.160 * * * \\
(0.059)\end{array}$ & $\begin{array}{l}1.173 * * * \\
(0.070)\end{array}$ \\
\hline Pampeana & $\begin{array}{c}0.017 \\
(0.054)\end{array}$ & $\begin{array}{c}1.017 \\
(0.055)\end{array}$ \\
\hline Patagónica & $\begin{array}{l}0.147 * * * \\
(0.053)\end{array}$ & $\begin{array}{l}1.158^{* * *} \\
(0.061)\end{array}$ \\
\hline Constante & $\begin{array}{l}-2.030^{* * *} \\
(0.106)\end{array}$ & $\begin{array}{l}0.131^{* * *} \\
(0.014)\end{array}$ \\
\hline $\begin{array}{l}\text { Observaciones } \\
\text { Pseudo } R^{2}\end{array}$ & $\begin{array}{c}92418 \\
0.272\end{array}$ & 92418 \\
\hline
\end{tabular}

Fuente: Elaboración propia a partir de datos de la Encuesta sobre Trabajo no Remunerado y Uso del Tiempo, año 2013.

Nota: entre paréntesis error estándar robusto. Significativo al: *** 1\%, ** 5\%, *10\%. Sin asterisco: no significativo.

La ventaja de identificar el grado de asociación de una variable con la pobreza, manteniendo el resto constante, se verifica, primero, en el resultado de la variable sexo 
del jefe que no resulta estadísticamente significativa. En otras palabras, las personas que viven en hogares con jefatura femenina tienen la misma probabilidad de ser pobres LIMTIP que aquellos en hogares comandados por hombres. Esto resultado contradice el Gráfico 3 en el que en la gran mayoría de los tipos de hogares definidos, la incidencia de la pobreza LIMTIP era mayor si su jefe de hogar era mujer. Pero una vez que se controla el comportamiento de la pobreza por otras variables, por ejemplo educación u ocupación del jefe de hogar, el efecto que era capturado en el análisis descriptivo por el género del jefe desaparece.

Entre los principales enemigos o batalladores en contra de la pobreza LIMTIP se encuentran los años de educación del jefe de hogar, la condición de ocupación adecuada del jefe de hogar (asalariado formal o cuentapropista profesional) y la presencia de adultos en el hogar, incluso de adultos mayores. Se observa una relación 4 a 1 en la probabilidad de ser pobre LIMTIP para aquellas personas en hogares cuyo jefe tiene un empleo en el sector informal o está inactivo. Pero esa probabilidad asciende a 13/1 cuando el jefe no tiene trabajo y está buscando uno activamente.

Los signos de los coeficientes correspondientes a las variables de cantidad de miembros del hogar no muestran variación según el género del miembro del hogar, pero sí según su edad. Así, los mayores de 17 años operan en contra de la pobreza, mientras que la presencia de niños y adolescentes se asocia con una mayor probabilidad de ser pobre. Entre los adultos mayores, la presencia de mujeres otorga una probabilidad menor de ser pobre LIMTIP que la presencia de adultos mayores varones, con una relación 4 a 1 si tienen entre 65 y 79 años, y 2 a 1 si tienen 80 años o más.

Por último, el modelo estimado refleja ciertas disparidades regionales dentro del país que resultan estadísticamente significativas. La mayoría de las regiones muestran una probabilidad mayor para sus habitantes de ser pobres LIMTIP, una vez controlando por el resto de las variables, que la región GBA excepto la región Pampeana.

Dado que la condición de pobreza LIMTIP es una combinación entre escasez de ingresos y déficit de tiempo, no resulta sencillo identificar con cuál de estos componentes se asocian más las variables explicativas. Es por eso que se presenta en la Tabla 6 el mismo modelo anterior para explicar la condición de pobreza en tiempo (columnas (1) y (2)) y pobreza en ingreso (columnas (3) y (4)). Una persona será 
considerada pobre en tiempo si vive en un hogar con déficit, lo cual equivale a decir que vive en un hogar con al menos un miembro deficitario.

\section{Tabla 6}

Modelo de regresión logística y Odds ratio de la condición de pobreza en tiempo y pobreza en ingreso

\begin{tabular}{|c|c|c|c|c|}
\hline & $\begin{array}{c}(1) \\
\text { Pobre en } \\
\text { tiempo }\end{array}$ & $\begin{array}{c}(2) \\
\text { Razón de } \\
\text { probabilidad }\end{array}$ & $\begin{array}{c}(3) \\
\text { Pobre en } \\
\text { ingreso }\end{array}$ & $\begin{array}{c}(4) \\
\text { Razón de } \\
\text { probabilidad }\end{array}$ \\
\hline Años de educación del jefe de hogar & $\begin{array}{r}0.002 \\
(0.004)\end{array}$ & $\begin{array}{r}1.002 \\
(0.004)\end{array}$ & $\begin{array}{l}-0.098 * * * \\
(0.006)\end{array}$ & $\begin{array}{l}0.907 * * * \\
(0.005)\end{array}$ \\
\hline $\begin{array}{l}\text { Jefe asalariado informal o cuentapropista no } \\
\text { profesional }\end{array}$ & $-0.083 *$ & $0.092 *$ & $1.431 * * *$ & $4.183^{* * *}$ \\
\hline & $(0.042)$ & $(0.039)$ & $(0.051)$ & $(0.215)$ \\
\hline Jefe ocupado (resto) & $\begin{array}{l}0.408 * * * \\
(0.069)\end{array}$ & $\begin{array}{l}1.504 * * * \\
(0.104)\end{array}$ & $\begin{array}{l}0.609 * * * \\
(0.097)\end{array}$ & $\begin{array}{l}1.838 * * * \\
(0.178)\end{array}$ \\
\hline Jefe inactivo & $\begin{array}{l}-1.293 * * * \\
(0.052)\end{array}$ & $\begin{array}{l}0.275 * * * \\
(0.014)\end{array}$ & $\begin{array}{l}1.760 * * * \\
(0.068)\end{array}$ & $\begin{array}{l}5.811^{* * *} \\
(0.395)\end{array}$ \\
\hline Jefe desocupado & $\begin{array}{l}-1.339^{* * *} \\
(0.091)\end{array}$ & $\begin{array}{l}0.262 * * * \\
(0.024)\end{array}$ & $\begin{array}{l}2.653 * * * \\
(0.103)\end{array}$ & $\begin{array}{l}14.190^{* * *} \\
(1.458)\end{array}$ \\
\hline Jefe varón & $\begin{array}{l}-0.258^{* * * *} \\
(0.042)\end{array}$ & $\begin{array}{c}0.772 * * * \\
(0.032)\end{array}$ & $\begin{array}{c}0.207 * * * \\
(0.050)\end{array}$ & $\begin{array}{c}1.230 * * * \\
(0.061)\end{array}$ \\
\hline Cantidad de niñas menores de 5 años & $\begin{array}{c}0.246 * * * \\
(0.041)\end{array}$ & $\begin{array}{c}1.279 * * * \\
(0.053)\end{array}$ & $\begin{array}{c}0.527 * * * \\
(0.045)\end{array}$ & $\begin{array}{c}1.694 * * * \\
(0.075)\end{array}$ \\
\hline Cantidad de niños menores de 5 años & $\begin{array}{c}0.613 * * * \\
(0.051)\end{array}$ & $\begin{array}{c}1.846^{* * *} \\
(0.944)\end{array}$ & $\begin{array}{c}0.392 * * * \\
(0.034)\end{array}$ & $\begin{array}{c}1.479 * * * \\
(0.050)\end{array}$ \\
\hline Cantidad de mujeres entre 5 y 17 años & $\begin{array}{l}0.722 * * * \\
(0.028)\end{array}$ & $\begin{array}{l}2.059^{* * *} \\
(0.058)\end{array}$ & $\begin{array}{l}0.486^{* * *} \\
(0.024)\end{array}$ & $\begin{array}{c}1.626^{* * * *} \\
(0.039)\end{array}$ \\
\hline Cantidad de varones entre 5 y 17 años & $\begin{array}{c}0.837 * * * \\
(0.026)\end{array}$ & $\begin{array}{c}2.309 * * * \\
(0.060)\end{array}$ & $\begin{array}{c}0.626^{* * * *} \\
(0.023)\end{array}$ & $\begin{array}{c}1.871 * * * \\
(0.042)\end{array}$ \\
\hline Cantidad de mujeres entre 18 y 64 años & $\begin{array}{c}0.217 * * * \\
(0.022)\end{array}$ & $\begin{array}{c}1.242 * * * \\
(0.028)\end{array}$ & $\begin{array}{c}-0.184 * * * \\
(0.026)\end{array}$ & $\begin{array}{c}0.832 * * * \\
(0.022)\end{array}$ \\
\hline Cantidad de varones entre 18 y 64 años & $\begin{array}{c}0.511 * * * \\
(0.027)\end{array}$ & $\begin{array}{c}1.666^{* * *} \\
(0.045)\end{array}$ & $\begin{array}{c}-0.251 * * * \\
(0.029)\end{array}$ & $\begin{array}{c}0.778 * * * \\
(0.023)\end{array}$ \\
\hline Cantidad de mujeres entre 65 y 79 años & $\begin{array}{c}0.140 * * \\
(0.064)\end{array}$ & $\begin{array}{l}1.151^{* *} \\
(0.073)\end{array}$ & $\begin{array}{c}-2.109 * * * \\
(0.131)\end{array}$ & $\begin{array}{c}0.121^{* * *} \\
(0.016)\end{array}$ \\
\hline Cantidad de varones entre 65 y 79 años & $\begin{array}{c}0.352 * * * \\
(0.056)\end{array}$ & $\begin{array}{l}1.421 * * \\
(0.079)\end{array}$ & $\begin{array}{c}-0.755^{* * *} \\
(0.163)\end{array}$ & $\begin{array}{c}0.470 * * * \\
(0.076)\end{array}$ \\
\hline Cantidad de mujeres de 80 años y más & $\begin{array}{c}0.468^{* * *} \\
(0.108)\end{array}$ & $\begin{array}{c}1.597 * * * \\
(0.173)\end{array}$ & $\begin{array}{c}-2.210^{* * *} \\
(0.158)\end{array}$ & $\begin{array}{c}0.110^{* * *} \\
(0.017)\end{array}$ \\
\hline Cantidad de varones de 80 años y más & $\begin{array}{c}0.259^{* * *} \\
(0.098)\end{array}$ & $\begin{array}{c}1.296^{* * *} \\
(0.126)\end{array}$ & $\begin{array}{c}-1.939 * * * \\
(0.193)\end{array}$ & $\begin{array}{c}0.144 * * * \\
(0.028)\end{array}$ \\
\hline NOA & $\begin{array}{c}0.041 \\
(0.040)\end{array}$ & $\begin{array}{c}1.042 \\
(0.041)\end{array}$ & $\begin{array}{c}0.003 \\
(0.055)\end{array}$ & $\begin{array}{c}1.003 \\
(0.055)\end{array}$ \\
\hline NEA & $\begin{array}{l}-0.059 \\
(0.044)\end{array}$ & $\begin{array}{c}0.943 \\
(0.041)\end{array}$ & $\begin{array}{c}0.302 * * * \\
(0.057)\end{array}$ & $\begin{array}{c}1.353^{* * *} \\
(0.077)\end{array}$ \\
\hline Cuyo & $\begin{array}{c}0.098 * * \\
(0.045)\end{array}$ & $\begin{array}{l}1.102 * * \\
(0.049)\end{array}$ & $\begin{array}{c}0.040 \\
(0.063)\end{array}$ & $\begin{array}{c}1.041 \\
(0.065)\end{array}$ \\
\hline Pampeana & $\begin{array}{l}-0.029 \\
(0.040)\end{array}$ & $\begin{array}{c}0.972 \\
(0.039)\end{array}$ & $\begin{array}{c}-0.049 \\
(0.057)\end{array}$ & $\begin{array}{c}0.952 \\
(0.055)\end{array}$ \\
\hline Patagónica & $\begin{array}{c}0.064 \\
(0.042)\end{array}$ & $\begin{array}{c}1.066 \\
(0.044)\end{array}$ & $\begin{array}{c}0.118^{* *} \\
(0.055)\end{array}$ & $\begin{array}{c}1.125^{* *} \\
(0.062)\end{array}$ \\
\hline Constante & $\begin{array}{c}-0.569 * * * \\
(0.085)\end{array}$ & $\begin{array}{c}0.566^{* * * *} \\
(0.482)\end{array}$ & $\begin{array}{c}-2.244 * * * \\
(0.111)\end{array}$ & $\begin{array}{c}0.106^{* * *} \\
(0.012)\end{array}$ \\
\hline $\begin{array}{l}\text { Observaciones } \\
\text { Pseudo } R^{2}\end{array}$ & $\begin{array}{c}92418 \\
0.212 \\
\end{array}$ & 92418 & $\begin{array}{c}92418 \\
0.258 \\
\end{array}$ & 92418 \\
\hline
\end{tabular}

Fuente: Elaboración propia a partir de datos de la Encuesta sobre Trabajo no Remunerado y Uso del Tiempo, año 2013.

Nota: entre paréntesis error estándar robusto. Significativo al: *** 1\%,** 5\%, * 10\%. Sin asterisco: no significativo. 
Se observa que el efecto positivo de los años de educación del jefe de hogar encontrado sólo tiene impacto sobre la condición de pobreza monetaria. Por otra parte, la condición laboral del jefe de hogar y su género tienen efectos contrarios: si el jefe trabaja en el sector informal, está desocupado o inactivo, la probabilidad de tener déficit de tiempo en el hogar es menor que si es asalariado formal o cuentapropista profesional. En cambio, la relación es totalmente adversa cuando la variable dependiente es la pobreza monetaria. Como el primer efecto es menor que el segundo, en términos generales toda ocupación distinta a la ideal (asalariado registrado o cuentapropista profesional) se asocia a una probabilidad mayor de pobreza LIMTIP.

Los habitantes de hogares con jefe varón se asocian con un menor déficit de tiempo pero una mayor escasez de ingresos, sin embargo la magnitud de los efectos son similares y es por eso que la variable sexo del jefe de hogar resulta estadísticamente no significativa en relación a la pobreza LIMTIP. Siguiendo con las variables demográficas, la presencia de niños y adolescentes favorecen a la condición de pobreza LIMTIP tanto en demanda de tiempo como de ingreso. Por su parte, los mayores de edad, si bien contribuyen a la pobreza en tiempo, retraen la pobreza monetaria. Hay una leve diferencia a favor de la presencia de mujeres en edades centrales porque cada mujer aporta una probabilidad menor a tener déficit de tiempo que cada varón $(0,24$ contra 0,66). Lo contrario ocurre en términos de pobreza monetaria.

Sin embargo, en todos los grupos de edad que generan fuerzas en diferentes sentidos en la pobreza monetaria y de tiempo, la magnitud del efecto a la baja sobre la pobreza monetaria supera la del efecto a favor del déficit de tiempo. Por lo tanto, la relación última de la cantidad de miembros mayores de edad con la pobreza LIMTIP es negativa.

\section{Consideraciones Finales}

Este documento introduce la dimensión temporal en la medición de pobreza con el objeto de analizar cómo se interrelacionan el mercado de trabajo, la estructura demográfica de los hogares y las políticas sociales con una perspectiva de género en la Argentina. Aplicando la metodología LIMTIP a los datos de la Encuesta sobre Trabajo no Remunerado y Uso del Tiempo del año 2013, se estimó la pobreza por ingreso corregida por el déficit de tiempo. Se encuentra que la pobreza LIMTIP afectaba en ese año a un quinto de la población, de los cuales 1 millón de personas no son pobres por ingresos pero sí pobres LIMTIP por sus déficits de tiempo. Ese millón de personas 
generalmente no es visibilizado en las medidas tradicionales y mucho menos tenido en cuenta en el diseño de políticas contra la pobreza.

La presencia de ocupados pobres LIMTIP sugiere que no basta con generar empleo para combatir la pobreza, también es preciso garantizar la creación de empleo de calidad con remuneraciones y jornadas laborales adecuadas. La pobreza LIMTIP también afecta a quienes no están ocupados, posiblemente por otros frentes más que por las largas jornadas laborales de las que están exentos; sea por enfrentar escasez de ingresos, importantes cargas demográficas y/o desigual distribución de las tareas domésticas.

Desde la perspectiva de género se ha encontrado evidencia de que la pobreza incide entre los hombres y las mujeres a niveles muy similares. Además, en un análisis condicional de la pobreza LIMTIP el género del jefe de hogar pierde significancia estadística cuando se incorporan controles sobre el tipo de ocupación y la educación del jefe. Todo esto sugiere que el patrón de mayor incidencia de pobreza LIMTIP en hogares con jefatura femenina responde a la diferencia en dotaciones entre jefes de diferente sexo y no a su condición de hombre o mujer.

Los miembros dependientes dentro del hogar, niños y adultos mayores, ejercen fuerzas contrarias respecto de la condición de pobreza LIMTIP. La presencia de niños y adolescentes está asociada con una mayor probabilidad de ser pobre LIMTIP, tanto por la demanda de ingresos como por los requerimientos de tiempo. Por otro lado, los adultos mayores, incluso los mayores de 80 años, muestran una relación negativa con el déficit de ambos componentes de la pobreza LIMTIP (ingreso y tiempo). Probablemente, el sistema de jubilaciones y pensiones que brinda cobertura a este grupo demográfico contribuye a la probabilidad de ser menos pobre por ingresos, a la vez que su presencia en el hogar significa una ayuda más que una carga.

Con todo, el diseño de políticas públicas orientadas a erradicar la pobreza debe considerar tres ejes fundamentales: mercado de trabajo, estructuras demográficas y protección social. El Estado debe propiciar una inserción laboral de calidad, con jornadas reguladas y salarios horarios más elevados expandiendo el empleo registrado. Estas políticas de crecimiento inclusivo deben garantizar que las mujeres puedan insertarse al mercado sin incurrir en un déficit de tiempo. Aun cuando no se encuentren diferencias en incidencia de la pobreza por discriminación, claramente los hogares con jefatura femenina están más desaventajados en término de pobreza LIMTIP. Por esto es 
preciso acompañar las políticas de empleo de calidad con servicios particulares pensados desde una perspectiva de género como son los servicios de cuidado.

\section{Referencias Bibliográficas}

Aguirre, R. y Ferrari, F. (2014). Las encuestas sobre uso del tiempo y trabajo no remunerado en América Latina y el Caribe. Caminos recorridos y desafíos hacia el futuro, Serie Asuntos de Género $\mathrm{N}^{\mathrm{0}} 122$, Santiago de Chile, Comisión Económica para América Latina y el Caribe (CEPAL). ISSN 1564-4170.

Antonopoulos, R., Esquivel, V., Masterson, T., y Zacharias, A. (2016). Measuring Poverty in the Case of Buenos Aires: Why Time Deficits Matter. Levy Economics Institute, Working Papers Series.

Arévalo, C. y Paz, J. (2015). Desigualdad entre géneros en el uso del tiempo total de trabajo (remunerado y no remunerado). Una exploración para la Ciudad de Buenos Aires. Lavboratorio, (26), 81-106.

Bardasi, E., y Wodon, Q. (2006). Working long hours and having no choice: Time poverty in Guinea. Feminist Economics, 16(3), 45-78.

Bloemen, H., Pasqua, S. y Stancanelli, E. (2010). An empirical analysis of the time allocation of Italian couples: are they responsive? Review of Economics of the Household, 8(3), 345-369.

Burchardt, T. (2008). Time and income poverty, CASE report 57 (London: LSE).

Carbajal, F. (2011). La consideración del uso de tiempo en el análisis de pobreza multidimensional. Tesis de Maestría, Facultad de Ciencias Económicas de la Universidad Nacional de La Plata, Buenos Aires, Argentina.

Dirección General de Estadísticas y Censos de la CABA (2007). El tiempo de trabajo total Mujeres y varones en la Ciudad de Buenos Aires. Informe de Resultado $\mathrm{N}^{\circ} 328$. Recuperado de: https://www.estadisticaciudad.gob.ar/eyc/wp-

content/uploads/2015/04/informe_328_encuesta_de_uso_del tiempo.pdf

Dirección Nacional de Encuestas de Hogares del INDEC (2003). Acerca del método utilizado para la medición de la pobreza en Argentina. Disponible en: http://www.indec.gov.ar/ftp/cuadros/sociedad/pobreza2.pdf. Última consulta: 4 de noviembre de 2016.

Esquivel, V. (2014). "La pobreza de ingreso y tiempo en Buenos Aires, Argentina Un ejercicio de medición de la pobreza para el diseño de políticas públicas". PNUD. Panamá, ISBN 9789962-688-27-3.

Ganem, J.; Giustiniani, P.; Peinado, G.; Ezpeleta, L; Gallo, F; Long, S., Sintes, P. y Andreozzi, L. (2014). "Los usos del tiempo en las ciudades de Rosario y de Buenos Aires. Estudio comparativo a través de las encuestas de uso del tiempo." XIX Jornadas "Investigaciones en la Facultad" de Ciencias Económicas y Estadística. Universidad Nacional de Rosario. ISSN N ${ }^{\circ}$ 1668-5008.

Grupo de Río (2007). Grupo de Expertos en Estadísticas de Pobreza. Compendio de mejores prácticas en la medición de la pobreza, Santiago de Chile.

McGinnity, F., y Russell, H. (2007). Work rich, time poor? Time-use of women and men in Ireland. Economic and Social Review, 38(3), 323. 
Martinez, J. (2005). La pieza que faltaba: uso del tiempo y regímenes de bienestar en América Latina. Nueva Sociedad, 199, 35-52.

Masterson, T. (2011). Quality of match for statistical matches used in the development of the Levy Institute Measure of Time and Income Poverty (LIMTIP) for Argentina, Chile, and Mexico. Levy Economics Institute of Bard College Working Paper, (692).

Masterson, T. (2012). Simulations of full-time employment and household work in the Levy Institute Measure of Time and Income Poverty (LIMTIP) for Argentina, Chile, and Mexico.

Masterson, T. (2014). Quality of statistical match and employment simulations used in the estimation of the Levy Institute Measure of Time and Income Poverty (LIMTIP) for South Korea, 2009. Levy Economics Institute, Working Paper, (793).

Merz, J., \& Rathjen, T. (2014). Time and income poverty: an interdependent multidimensional poverty approach with German time use diary data. Review of Income and Wealth, 60(3), 450479.

Naciones Unidas (NU, 2015). Seguimiento de los resultados de la Cumbre del Milenio. A/69/L.85, 12 de agosto.

Öneş, U., Memiş, E., y Kızılırmak, B. (2013). Poverty and intra-household distribution of work time in Turkey: Analysis and some policy implications. In Women's Studies International Forum (Vol. 41, pp. 55-64). Pergamon.

Ravallion, M. (1999). Las líneas de pobreza en la teoría y en la práctica. Jorge Carpió e Irene Novacovsky (comp), De igual a igual. El desafío del Estado ante los nuevos problemas sociales, Editorial Fondo de Cultura Económica y Secretaría de Desarrollo Social de la Nación, Argentina. Reimers, Fernando (1999a), Educación, Pobreza y Desigualdad en América Latina, Mimeo, México.

Rupnik, A. y Colombo, P. (sin fecha). Las mujeres cuentan. Contemos el trabajo de las mujeres. Investigación sobre distribución del uso del tiempo entre las mujeres de la Ciudad de Buenos Aires. Boletín del Sindicato de Amas de Casa de la República Argentina. Disponible en: http://aaps.org.ar/pdf/rupnik_colombo.pdf. Última consulta: 4 de noviembre de 2016.

UNIFEM, Fondo de desarrollo de las Naciones Unidas para la Mujer (2005). El Progreso de las Mujeres en el Mundo, Mujeres, Trabajo y Pobreza. New York, ISBN: 1-932827-26-9.

Vickery, C. (1977). The Time-Poor: A New Look at Poverty. The Journal of Human Resources, 12, 27-48. Nro.1. Recuperado de: http://www.jstor.org/stable/145597 doi:1

Zacharias, A., Antonopoulos, R. y Masterson, T. (2012): Why Time Deficits Matter: Implications for the Measurement of Poverty. Research Project Report. Annandale-OnHudson, N.Y.: Levy Economics Institute of Bard College.

Zacharias, A., Antonopoulos, R. y Masterson, T. (Sin fecha): Time Deficits and the Measurement of Income Poverty: Methodology and Evidence from Latin America. 